ARTICLE

DOI: $10.1038 /$ s41467-017-00654-w

\title{
Uncontrolled angiogenic precursor expansion causes coronary artery anomalies in mice lacking Pofut1
}

Yidong Wang ${ }^{1}$, Bingruo $\mathrm{Wu}^{1}$, Pengfei $\mathrm{Lu}^{1}$, Donghong Zhang ${ }^{1}$, Brian $\mathrm{Wu}^{1}$, Shweta Varshney ${ }^{2}$, Gonzalo del Monte-Nieto (10 3,4, Zhenwu Zhuang5, Rabab Charafeddine ${ }^{6}$, Adam H. Kramer ${ }^{6}$, Nicolas E. Sibinga (1) ${ }^{7}$, Nikolaos G. Frangogiannis ${ }^{8}$, Richard N. Kitsis (1) ${ }^{9}$, Ralf H. Adams (1) ${ }^{10}$, Kari Alitalo ${ }^{11}$, David J. Sharp ${ }^{6}$, Richard P. Harvey ${ }^{3,4,12}$, Pamela Stanley (i) ${ }^{2} \&$ Bin Zhou (iD ${ }^{1,13}$

Coronary artery anomalies may cause life-threatening cardiac complications; however, developmental mechanisms underpinning coronary artery formation remain ill-defined. Here we identify an angiogenic cell population for coronary artery formation in mice. Regulated by a DLL4/NOTCH1/VEGFA/VEGFR2 signaling axis, these angiogenic cells generate mature coronary arteries. The NOTCH modulator POFUT1 critically regulates this signaling axis. POFUT1 inactivation disrupts signaling events and results in excessive angiogenic cell proliferation and plexus formation, leading to anomalous coronary arteries, myocardial infarction and heart failure. Simultaneous VEGFR2 inactivation fully rescues these defects. These findings show that dysregulated angiogenic precursors link coronary anomalies to ischemic heart disease.

\footnotetext{
${ }^{1}$ Departments of Genetics, Pediatrics, and Medicine (Cardiology), Wilf Cardiovascular Research Institute, Albert Einstein College of Medicine, Bronx, New York 10461, USA. ${ }^{2}$ Department of Cell Biology, Albert Einstein College of Medicine, Bronx, NY 10461, USA. ${ }^{3}$ Developmental and Stem Cell Biology Division, Victor Chang Cardiac Research Institute, Darlinghurst, NSW 2010, Australia. ${ }^{4}$ St. Vincent's Clinical School, University of New South Wales, Kensington, NSW 2052, Australia. ${ }^{5}$ Department of Medicine, Yale University, New Haven, Connecticut 06510, USA. ${ }^{6}$ Department of Physiology and Biophysics, Albert Einstein College of Medicine, Bronx, New York 10461, USA. ${ }^{7}$ Departments of Medicine, Developmental and Molecular Biology, Wilf Cardiovascular Research Institute, Albert Einstein College of Medicine, Bronx, New York 10461, USA. ${ }^{8}$ Departments of Medicine, Microbiology and Immunology, Wilf Cardiovascular Research Institute, Albert Einstein College of Medicine, Bronx, New York 10461, USA. ${ }^{9}$ Departments of Medicine and Cell Biology, Wilf Cardiovascular Research Institute, Albert Einstein College of Medicine, Bronx, New York 10461, USA. ${ }^{10}$ Department of Tissue Morphogenesis, Max-Planck-Institute for Molecular Biomedicine, Röntgenstraße 20, and Faculty of Medicine, University of Münster, 48149 Münster, Germany. ${ }^{11}$ Wihuri Research Institute, Biomedicum Helsinki, Haartmaninkatu 8, Fl-00290 Helsinki, Finland. ${ }^{12}$ School of Biotechnology and Biomolecular Science, University of New South Wales, Kensington, NSW 2052, Australia. ${ }^{13}$ Department of Cardiology, The First Affiliated Hospital of Nanjing Medical University, Nanjing, Jiangsu 210029, China. Correspondence and requests for materials should be addressed to B.Z. (email: bin.zhou@einstein.yu.edu)
} 
C oronary arteries provide nutrients and oxygen to cardiac muscle, and are therefore essential for heart functions ${ }^{1,2}$. Consisting of three tissue layers (an inner layer of endothelium, middle layer of vascular smooth muscle and outer layer of fibroblasts), coronary arteries are formed by a tightly-regulated and complex process, disruption of which may cause coronary artery anomalies, leading to sudden cardiac death ${ }^{3,4}$, myocardial infarction $^{5}$ or heart failure ${ }^{6}$. In mice, development of coronary vessels begins with the formation of an endothelial plexus by precursor cells arising in ventricular endocardium ${ }^{7}$, sinus venous endocardium ${ }^{8}$, and proepicardium/epicardium ${ }^{9}$. Regardless of their origins, these progenitors invade the myocardium and proliferate to form coronary plexuses (primitive coronary vessels) by vasculogenesis (de novo vessel formation). The deeper part of the coronary plexus is specified to an arterial fate and coalesces then recruits pericytes to become mature coronary arteries with a smooth muscle media ${ }^{10,11}$. Despite the profound importance of coronary arteries, the characteristics of coronary angiogenic precursor cells and the molecular mechanisms that drive them to form coronary arteries remain poorly understood.

NOTCH signaling regulates multiple cellular functions in heart development and disease ${ }^{12,13}$. Notch genes encode transmembrane receptors (NOTCH1-4) that interact with membranebound ligands of the Delta (DLL1,3,4) and Jagged (JAG1,2) family ${ }^{14}$. The binding of NOTCH ligands is regulated by protein $\mathrm{O}$-fucosyltransferase 1 (POFUT1). Bound ligand triggers cleavage of the associated receptor and then release of its intracellular domain (NOTCH intracellular domain, which shuttles to the nucleus where it forms a transcriptional complex with RBPjk and activates the expression of target genes ${ }^{15}, 16$. NOTCH signaling functions as a key regulator of angiogenesis outside of the heart in physiological and pathological conditions ${ }^{17-19}$, in particular arterial-venous specification ${ }^{20,21}$, tip and stalk cell selection during sprouting angiogenesis ${ }^{22-26}$, proliferation and survival of endothelial cells ${ }^{27}$, and smooth muscle recruitment and differentiation ${ }^{28-32}$. Whether NOTCH signaling plays similar roles in coronary artery development is incompletely understood ${ }^{31}$. This is partly due to lack of functional definition of progenitor cells for coronary arteries.

Here we functionally characterize a coronary angiogenic precursor population, which expresses high levels of VEGFR3. We show that POFUT1, a regulator of NOTCH ligand binding, regulates this precursor pool through DLL4/NOTCH1/VEGF signaling. Disruption of Pofut1 in ventricular endocardium results in structural coronary artery anomalies and early-onset ischemic heart disease due to insufficient coronary oxygen perfusion. Loss of Pofut1 promotes proliferation of angiogenic precursors to form excessive coronary plexuses, which fail to undergo proper arteriogenesis.

\section{Results}

Coronary artery anomalies in mice lacking endocardial Pofut1. To investigate functions of POFUT1, an upstream modulator of ligand-induced NOTCH signaling ${ }^{33-35}$, in heart development, we deleted Pofut1 in individual cardiac cell lineages by crossing floxed Pofut1 mice with tissue-specific Cre lines including Tnt ${ }^{\text {Cre }}$ for myocardium, $\mathrm{Tb} \times 18^{\mathrm{Cre}}$ for epicardium, $\mathrm{Sm} 22 \alpha^{\mathrm{Cre}}$ for vascular smooth muscle, Mef2 2 Cre for secondary heart field tissue, Tie $1^{\mathrm{Cre}}$ for vascular endothelium, and $\mathrm{Nfatc1}^{\mathrm{Cre}}$ for cardiac endocardium. Mice with Pofut1 deletion in myocardium, epicardium, vascular smooth muscle, or secondary heart field were healthy and lived to adulthood (Supplementary Table 1). In contrast, deletion of Pofut1 in endocardium (Nfatc1 ${ }^{\text {Cre }} ;$ Pofut $1^{\mathrm{f} / \mathrm{f}}$, hereafter Pofut $1^{\mathrm{cKO}}$ ) (Supplementary Fig. 1) resulted in death of $\sim 80 \%$ Pofut ${ }^{c K O}$ mice by 4 months of age (Fig. 1a). Nearly $40 \%$ of endocardial
Pofut $1^{\mathrm{cKO}}$ died of heart failure at a young age between postnatal day (P) 40 and P120, indicated by dilated heart (Fig. 1b), increased heart/body weight (Fig. 1c), and impaired cardiac function, including increased left ventricular volume and reduced fractional shortening as well as ejection fraction at P60 (Fig. 1d). There was also elevated $M y h 7$ and decreased Myh6 expression in diseased hearts (Fig. 1e), a 'molecular switch' indicative of heart failure $^{36}$. Pathology evaluations by Hematoxylin/Eosin and Sirius Red staining revealed that diseased hearts had enlarged chambers, sub-endocardial fibrosis, and grossly dilated coronaries at P60 (Supplementary Fig. 2a-e). Another $40 \%$ of Pofut $1^{\mathrm{cKO}}$ mice died at an even younger stage between P19 and P40 with severe myocardial infarction, as evidenced by large areas of myocardial necrosis and fibrosis (Fig. 1a, f, g, and Supplementary Fig. 2f). Of note, before myocardial infarction, the myocardium of Pofut $1^{\text {cKO }}$ mice was already severely hypoxic (Fig. 1h) and some cardiomyocytes underwent apoptosis, whereas no apoptotic cells were found in the myocardium of control hearts (Supplementary Fig. 2g). These observations indicated poor coronary perfusion in Pofut $1^{\text {cKO }}$ hearts. Micro-computed tomography confirmed dysfunctional coronary arteries by revealing hypoplastic main coronary arteries and aneurysms (Fig. 1i, Supplementary Fig. 3a). Quantitative analysis of coronary arterial trees showed that Pofut $1^{\mathrm{cKO}}$ hearts had a decreased number of middle- to large-size arteries, and an increased number of small arteries (Supplementary Fig. 3b). Antibody staining for PECAM1, a marker of vascular endothelium showed increased coronary plexus formation in Pofut $1^{\mathrm{cKO}}$ hearts at P15 (Fig. 1j, Supplementary Fig. 2h). These findings demonstrate that endocardial loss of Pofut 1 causes coronary artery anomalies, leading to early-onset ischemic heart disease.

POFUT1 is required for coronary arteriogenesis. Coronary structural anomalies have a fetal origin. We therefore examined the embryonic coronary arteries of control and Pofut $1^{\mathrm{cKO}}$ hearts using coronary arteriography with fluorescent dye. The results showed that Pofut $1^{c K O}$ hearts had already developed hypoplastic main coronary arteries at embryonic day (E) 16.5 (Fig. 2a). Co-immunostaining with antibodies for PECAM1 and DLL4 (predominantly expressed by arterial endothelium) confirmed that Pofut $1^{\mathrm{cKO}}$ hearts had underdeveloped main coronary arteries in the outer myocardium close to the epicardium. In addition, the density of small coronary arteries in the inner myocardium close to the endocardium was significantly increased (Fig. 2b). Coimmunostaining of Isolectin B4 (IB4) expressed in endothelium and SMMHC (smooth muscle myosin heavy chain) or $\alpha \mathrm{SMA}$ (alpha-smooth muscle actin) for vascular smooth muscle revealed that Pofut $1^{\mathrm{cKO}}$ coronary arteries had poor smooth muscle media (Fig. 2c, Supplementary Fig. 4a). Pofut $1^{\mathrm{cKO}}$ hearts at E16.5 also had decreased expression of smooth muscle marker genes (Smmhc, Sm $22 \alpha$ and $\alpha S M A)$ and NOTCH pathway genes (Dll4, Jag1, Notch3, Notch4, HeyL and Hes5) (Supplementary Fig. 4b). Additionally, expression of cell junction genes (Itgb3, Cx37 and $C \times 40)$ involved in endothelium-pericyte adhesion was decreased in E16.5 Pofut $1^{\mathrm{cKO}}$ hearts, whereas expression of genes involved in pericyte differentiation (PDGFB, TIE-Angiopoietin and TGFB pathway genes) or arterial and venous endothelial markers was not affected by deletion of Pofut1 (Supplementary Fig. 5). Consistently, functional assays using E11.5 ventricle explant culture suggested defective attachment of SM $22 \alpha^{+}$pericytes to the extruding endocardium-derived endothelial cells, which were genetically labeled by GFP using the $N f a t c 1^{C r e}$ driver (Fig. 2d). The structural coronary artery anomalies in Pofut $1^{\mathrm{cKO}}$ hearts led us to examine coronary perfusion. Hypoxia probe labeling showed that the inner myocardium of E16.5 control hearts was 
a

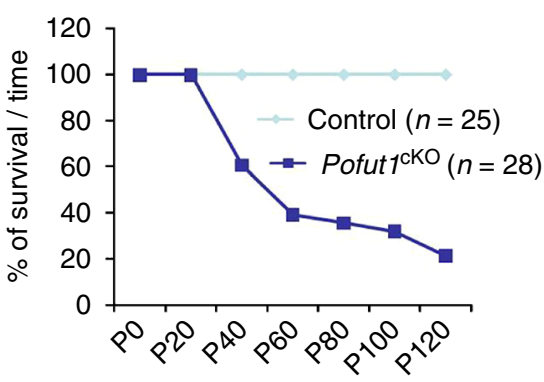

d

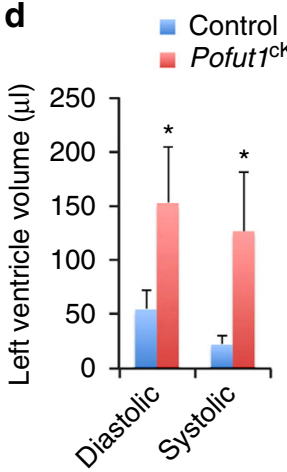

g

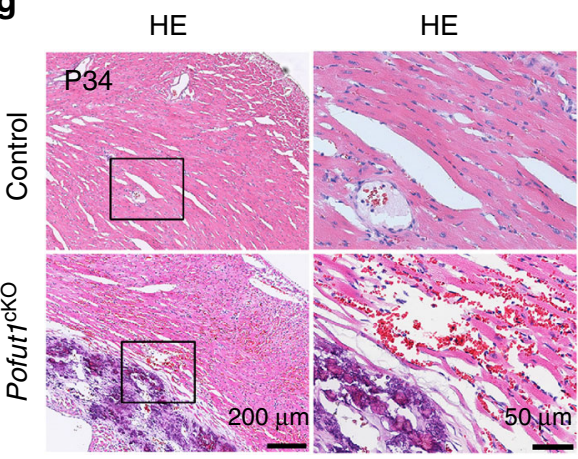

i
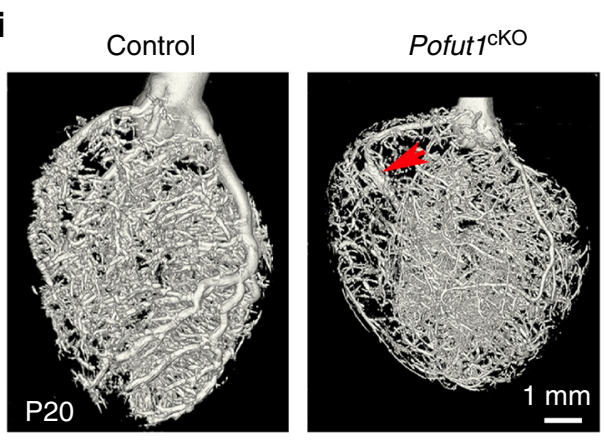

b

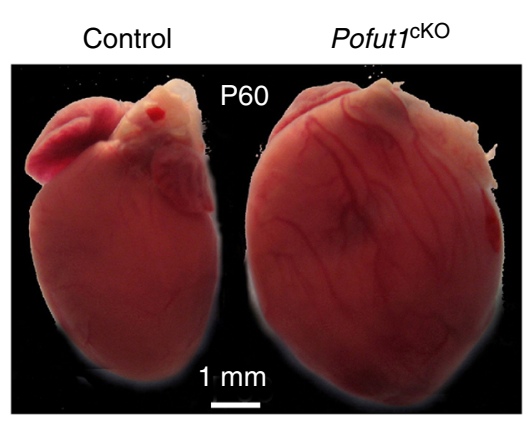

e
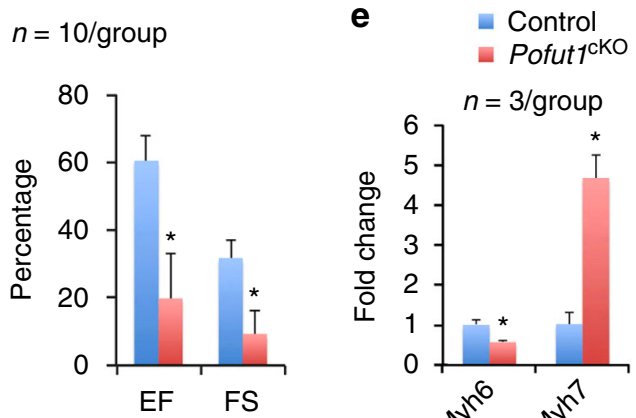

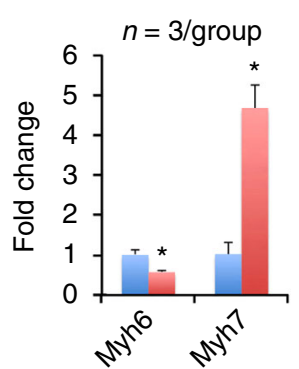

C

C
$\quad$ Control
Pofut ${ }^{\mathrm{cKO}}$

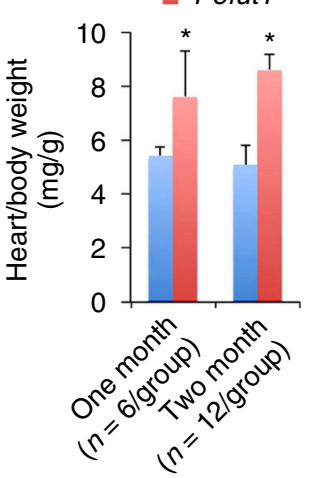

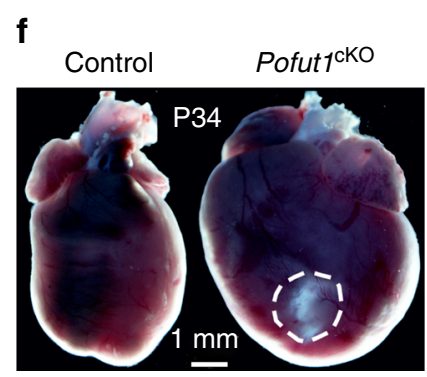

h

Sirius red

Hypoxia probe

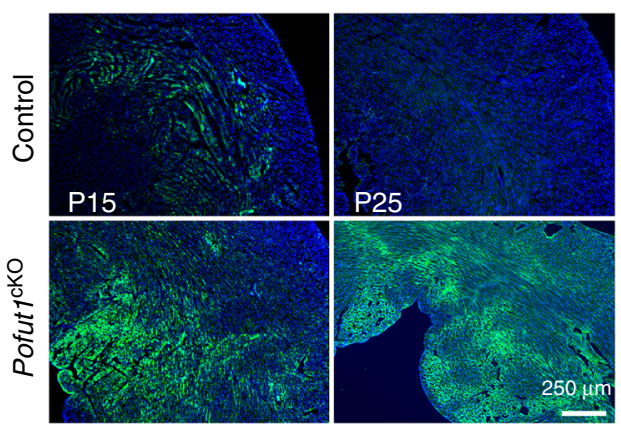

j

Control

Pofut ${ }^{\mathrm{ckO}}$
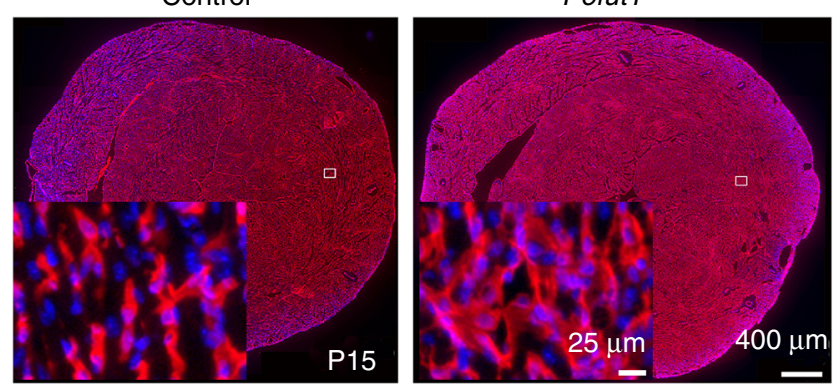

Fig. 1 Loss of Pofut1 causes coronary artery anomalies and ischemic heart disease. a Survival curve shows $\sim 80 \%$ Nfatc $7^{\mathrm{Cre}} ; \mathrm{Pofut} \mathrm{f}^{\mathrm{f} / \mathrm{f}}$ (Pofut $\mathrm{\tau}^{\mathrm{CKO}}$ ) mice die by P120. b Gross view of a dilated P60 Pofut $7{ }^{\mathrm{K} O}$ heart. $\mathbf{c}$ Heart/body ratio. ${ }^{\star} p<0.01$. d Echocardiography showing reduced cardiac functions in P60 Pofut $7 \mathrm{cKO}$ heart. ${ }^{\star} p<0.001$. e qPCR showing reversed expression changes of Myh6 and Myh7 in P21 Pofut $7{ }^{\mathrm{K} K O}$ hearts. ${ }^{\star} p<0.01$. f Gross view of a large myocardial infarct (circle) at the apex of a P34 Pofut $7^{\mathrm{CKO}}$ heart. $\mathbf{g}$ Cardiac histology showing cardiac necrosis and fibrosis in a P34 Pofut ${ }^{\mathrm{CKO}}$ heart. $\mathbf{h}$ Hypoxia staining showing expansion of inner myocardial hypoxia in young Pofut $7^{\mathrm{K} O}$ mice. i MicroCT shows coronary anomalies with hypoplastic main arteries and aneurisms (arrow). j PECAM1 staining shows increased primitive coronary plexuses in a P15 Pofut7 ${ }^{\mathrm{K} O}$ heart. All bar charts represent mean \pm s.d

hypoxic and this hypoxic region was largely expanded in Pofut $1^{\text {cKO }}$ hearts (Fig. 2e, f). This result suggests that the increased coronary plexuses in the inner myocardium of Pofut $1^{\mathrm{cKO}}$ hearts are dysfunctional and immature vessels. Consistent with the hypoxic finding, VEGFA antibody staining showed that relatively high levels of VEGFA were located in the inner myocardium of control hearts and this VEGFA-positive region was dramatically expanded in Pofut $1^{\mathrm{cKO}}$ hearts (Fig. $2 \mathrm{~g}, \mathrm{~h}$ ).

To determine whether endocardial deletion of Pofut1 affected formation of coronary veins and cardiac lymphatic vessels, we 


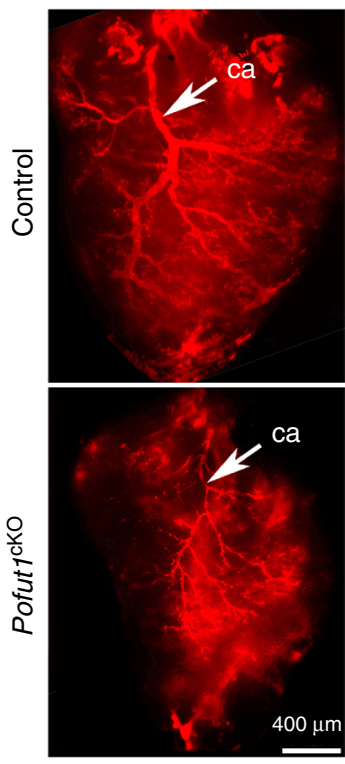

c

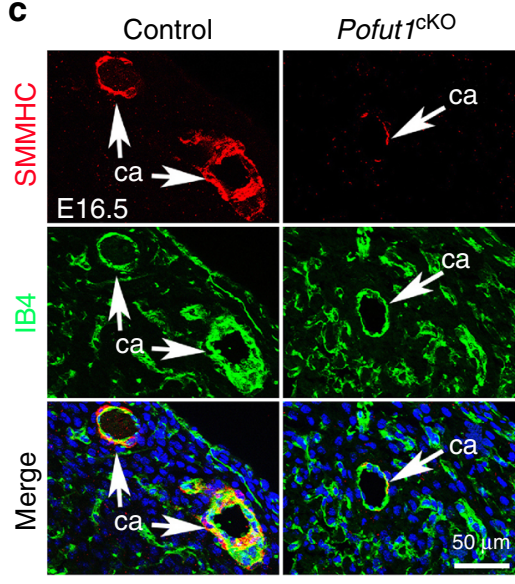

b

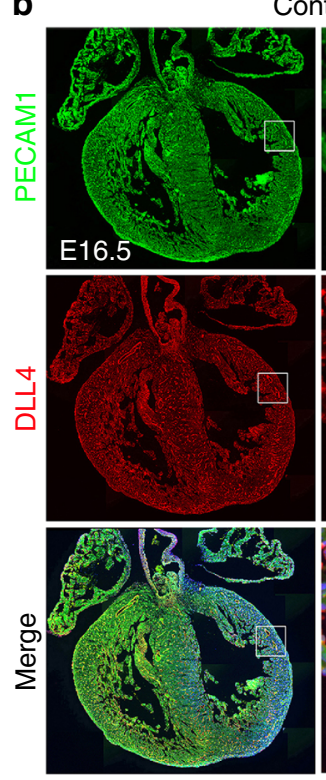

d

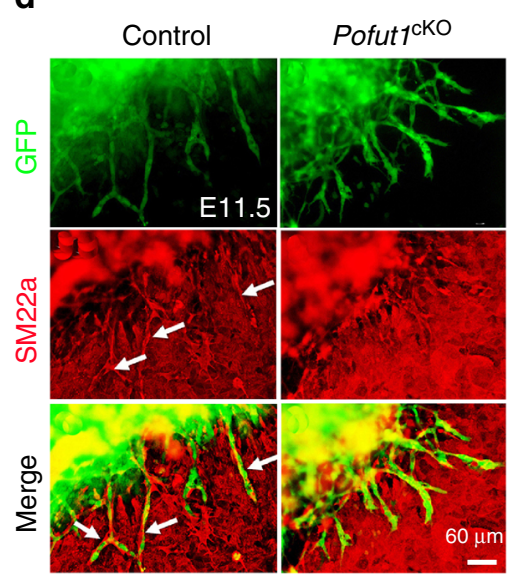

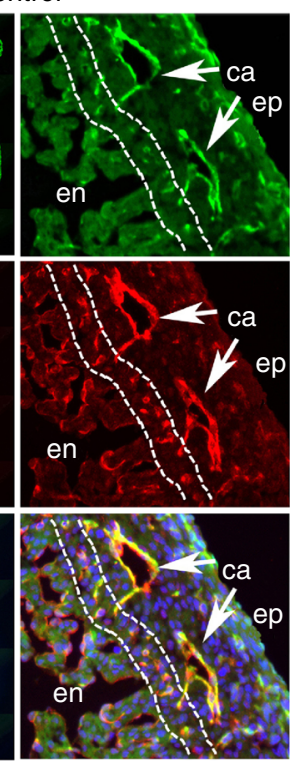

a
Pofut $1^{\text {cko }}$

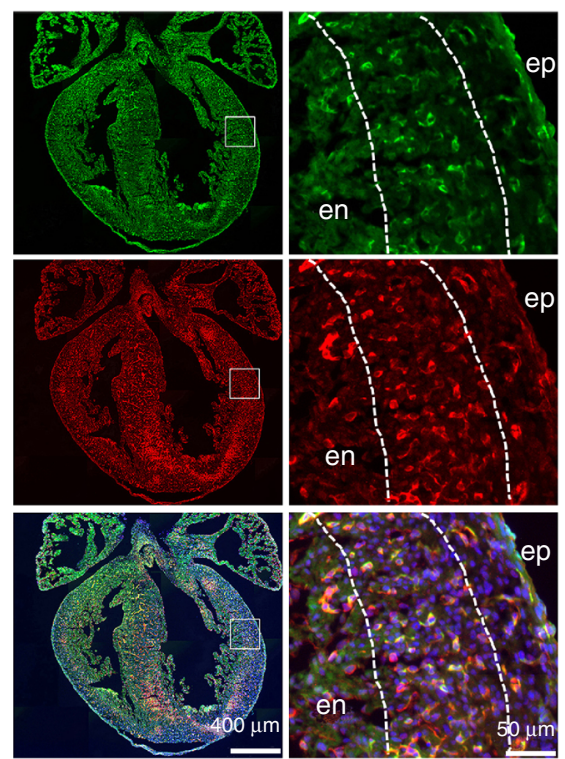

e
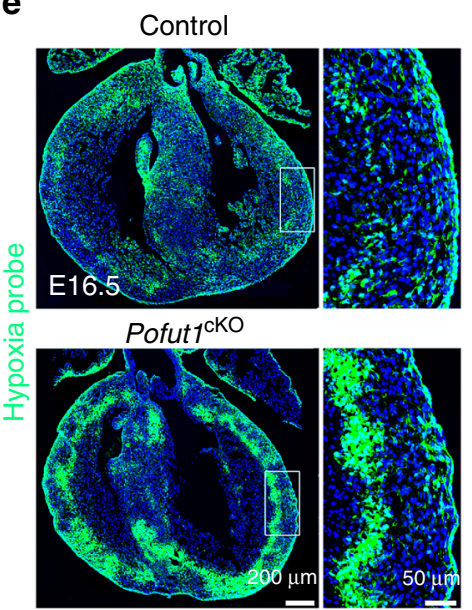

f Control g

- Pofut ${ }^{\mathrm{ckO}}$

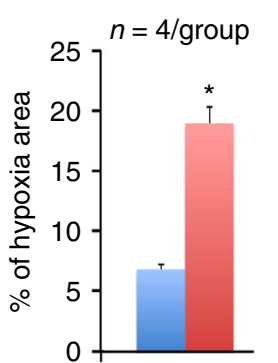

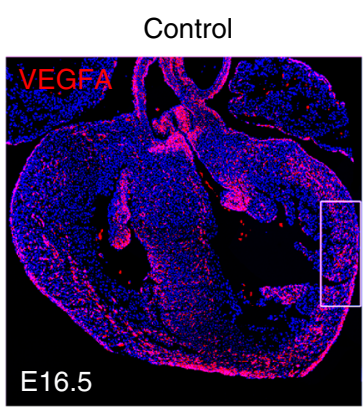

h

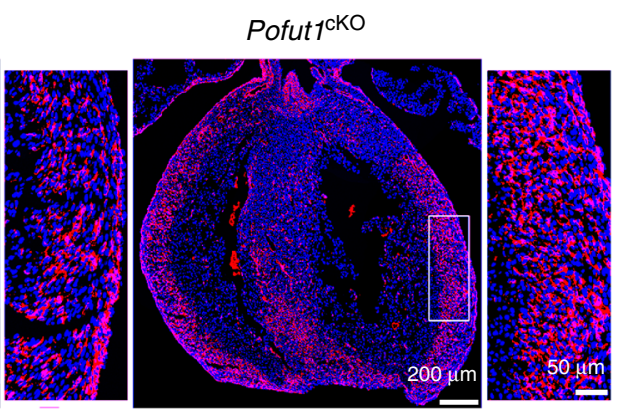

- Control

$$
\text { - Pofut }{ }^{\mathrm{CKO}}
$$

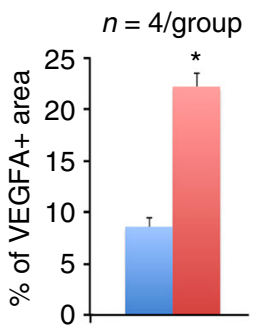

Fig. 2 POFUT1 is required for coronary artery formation. a Coronary arteriography using red fluorescent dye showing underdeveloped coronary arteries (ca,

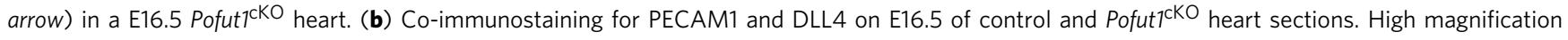
images on right showing boxed regions in the individual left ventricles with smaller main arteries (arrows) and expansion of arterial plexus in the inner myocardium (between dotted lines) in a Pofut $7^{\mathrm{CKO}}$ heart. c SMMHC and Isolectin-B4 (IB4) co-immunostaining showing hypoplastic major coronary arteries with thin smooth muscle layer (arrow) in a Pofut7 ${ }^{\mathrm{CKO}}$ heart. d E11.5 ventricular explant culture (GFP labels ventricular endocardial-derived plexus, red fluorescence labels pericytes using SM22 $\alpha$ antibodies) showing poor pericyte attachment to the plexus in a Pofut $7^{c K O}$ explant. e, f Representative hypoxia probe staining and quantifications show expansion of inner myocardial hypoxic zone in an E16.5 Pofut ${ }^{c K O}$ heart. ${ }^{\star} p<0.01$. g, h Representative VEGFA immunostaining and quantifications show expansion of VEGFA expression in the myocardial wall of an E16.5 Pofut7 ${ }^{\mathrm{KKO}}$ heart.

${ }^{*} p<0.01$. en/ep endocardium/epicardium 
a

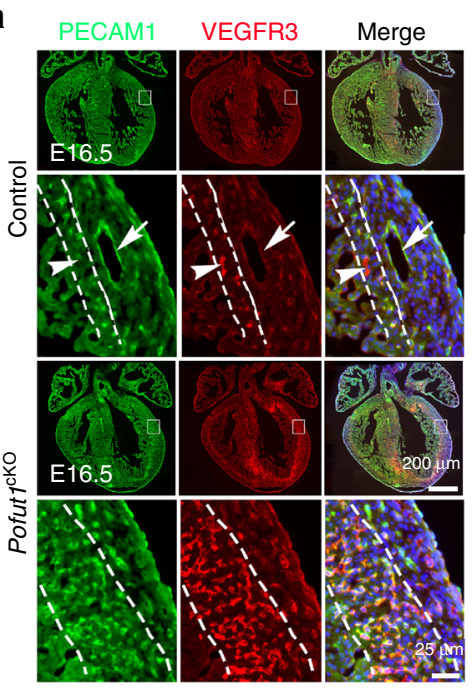

e
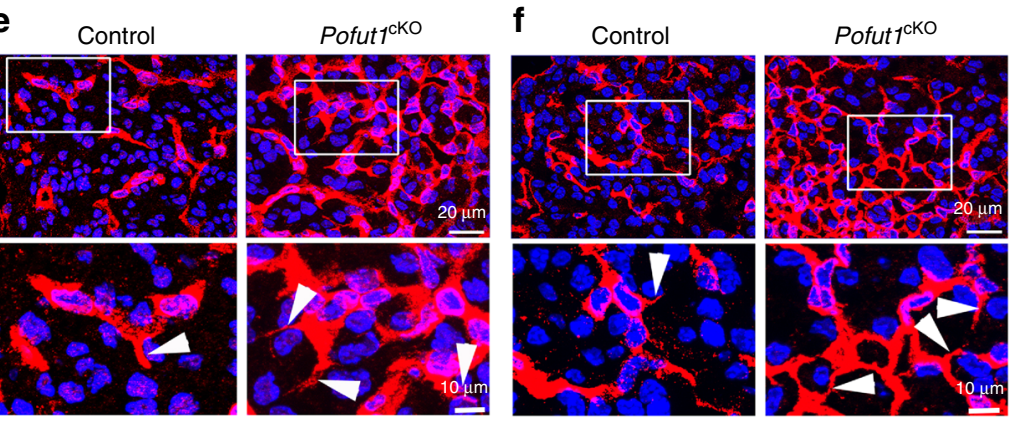

DLL4/DAPI
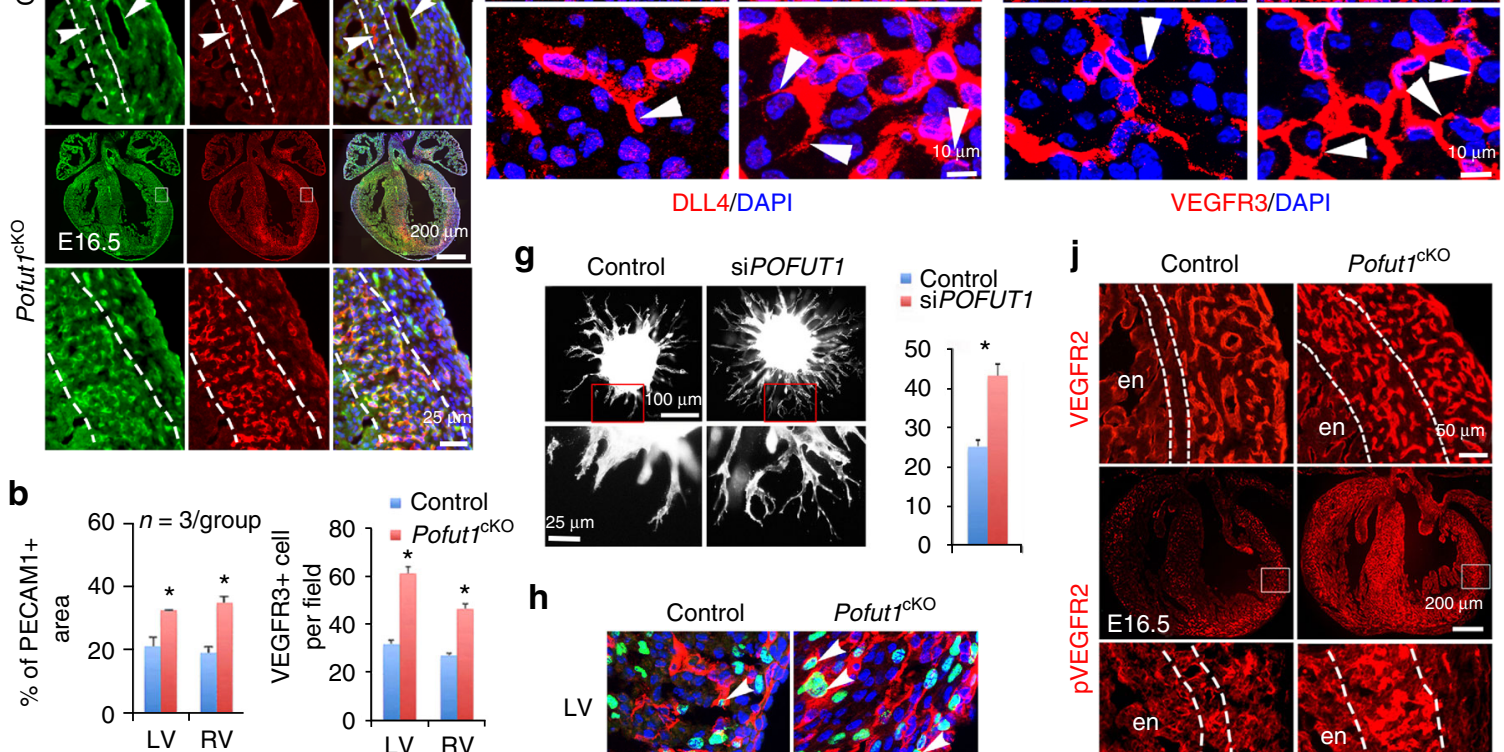

h

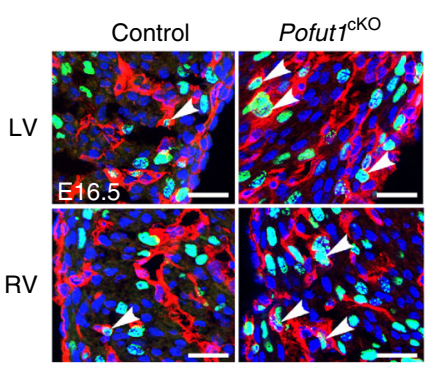

PECAM1/EdU/DAPI
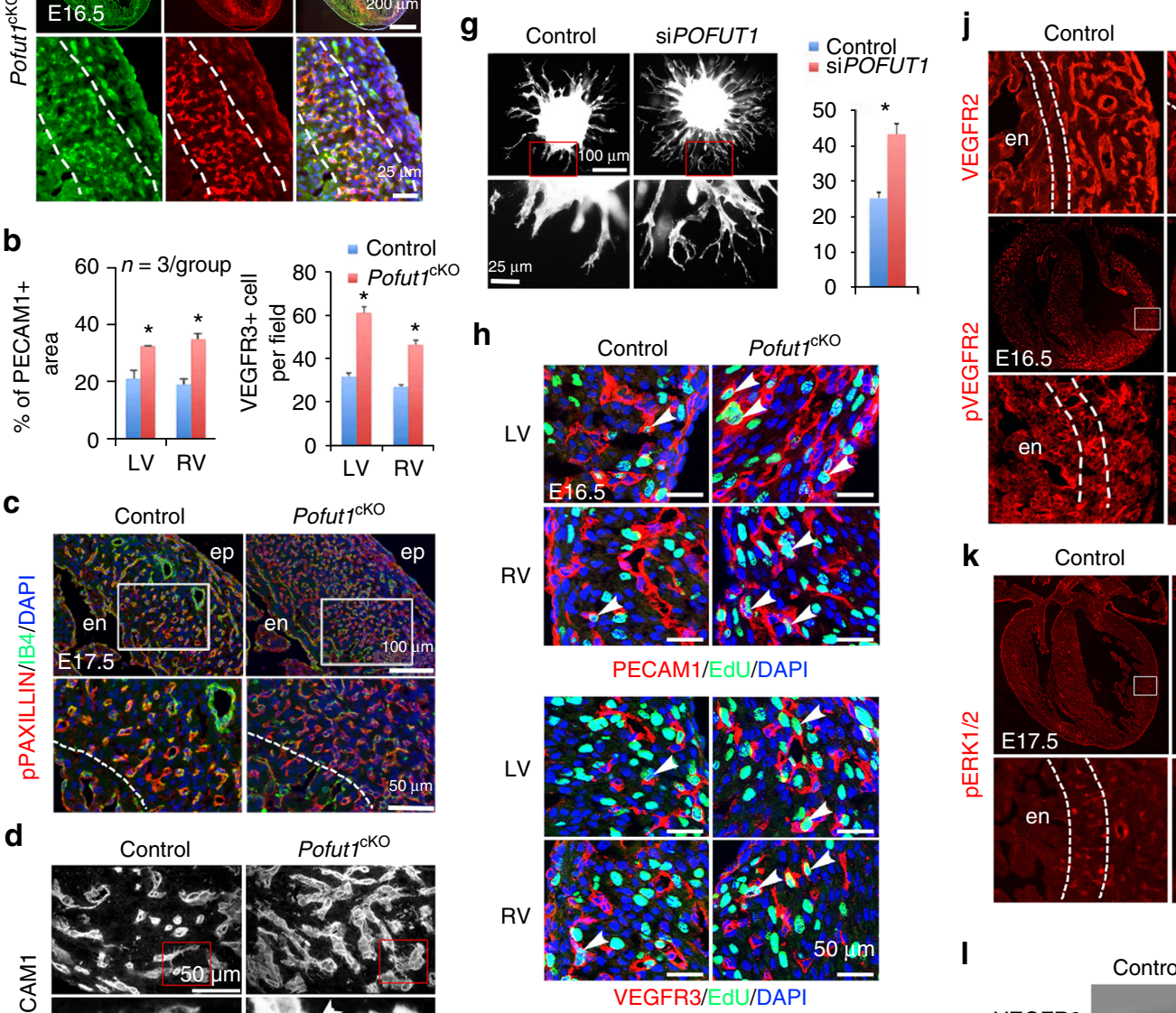

Pofut $1^{\text {cko }}$

d

$$
\text { Control Pofut } 1^{\mathrm{ckO}}
$$

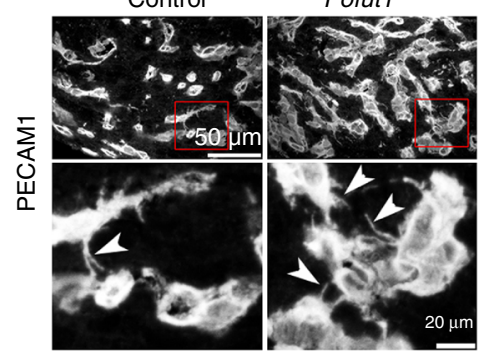

i

- Control

- Pofut ${ }^{\text {cko }}$
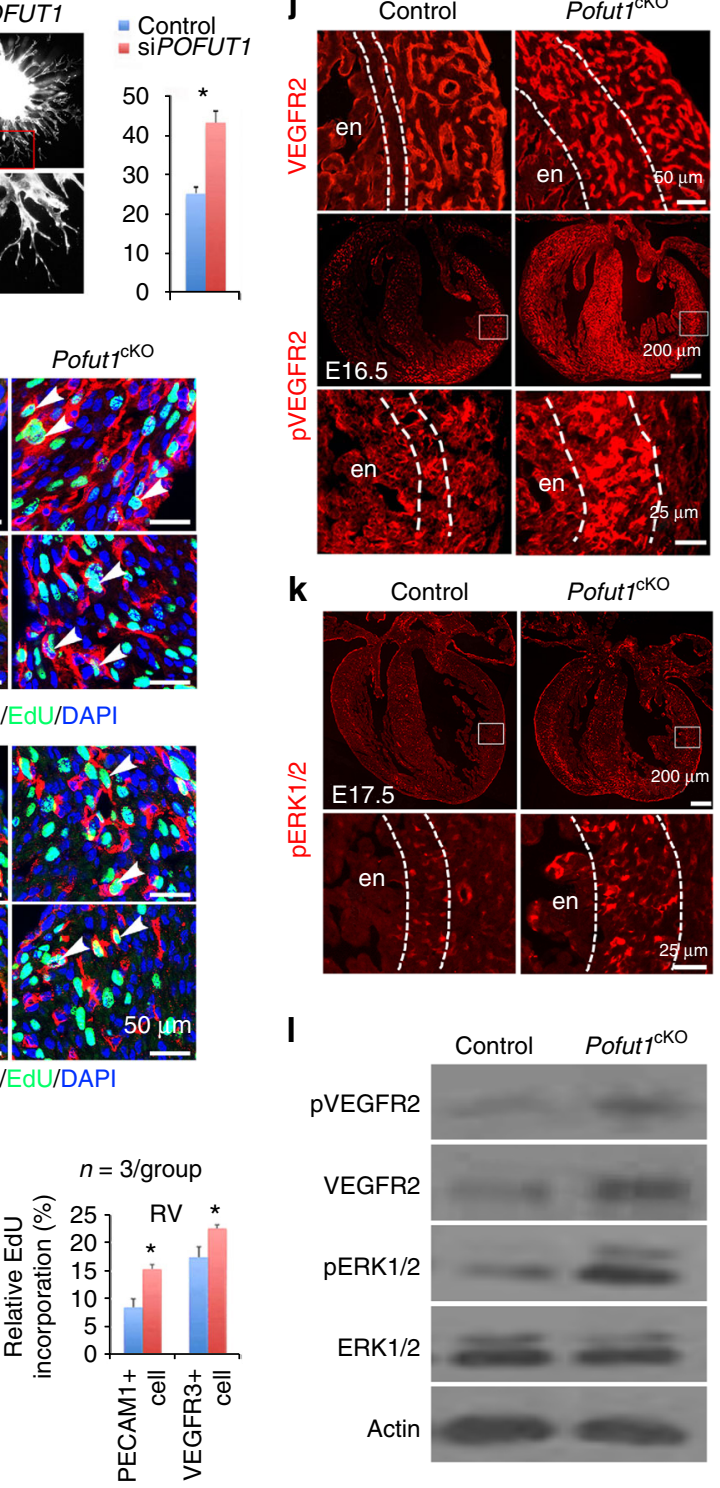

k

Control

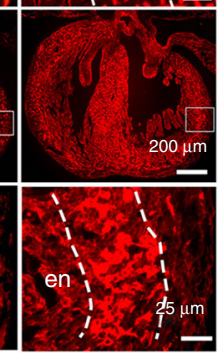

k

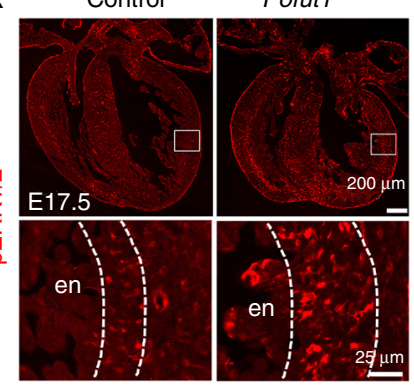

I

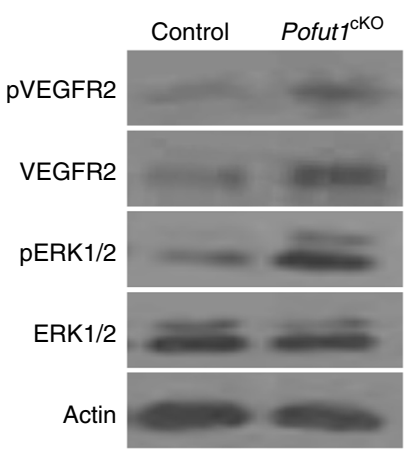

Fig. 3 POFUT1 regulates angiogenic functions and proliferation of coronary angiogenic cells. a, b PECAM1/VEGFR3 co-staining of E16.5 heart sections shows VEGFR3 ${ }^{\text {high }}$ coronary angiogenic cells (arrowhead) in the inner myocardium (between dotted lines) and VEGFR3 ${ }^{\text {low }}$ coronary arteries (arrow). b Quantification of PECAM1+ areas and the number of VEGFR3 ${ }^{\text {high }}$ cells. ${ }^{*} p<0.01$. c IB4 and pPAXILLIN co-staining of E17.5 heart sections. en/ ep, endocardium/epicardium. d PECAM1 immunostaining showing increased tip-like cells (arrowhead) in myocardium of E16.5 Pofut 7 CKO embryos. ${ }^{\star} p<$ 0.01. e, $\mathbf{f}$ Immunostaining of DLL4 and VEGFR3. $\mathbf{g}$ Spheroid sprouting assay with phalloidin staining showing increased sprouts by inhibition of POFUT1 using shRNA. Eight spheroids per group, $n=3,{ }^{\star} p<0.001$. h, i EdU labeling for proliferative PECAM1+ or VEGFR3+ cells (arrowheads). ${ }^{\star} p<0.05$. All bar charts represent mean $\pm \mathrm{SD}$. $\mathbf{j}$ immunostaining of VEGFR2 and pVEGFR2. $\mathbf{k}$ Immunostaining of pERK1/2. I Western blot analysis of pVEGFR, VEGFR2, pERK1/2, and ERK1/2 expression in E16.5 control and Pofut7 ${ }^{\mathrm{CKO}}$ hearts 
performed immunostaining for venous marker EMCN and lymphatic maker LYVE1, respectively. The results showed that some small intramyocardial coronary plexuses in Pofut $1^{\mathrm{cKO}}$ embryos were positive for EMCN while coronary veins on the heart surface were comparable between control and Pofut $1^{\mathrm{CKO}}$ embryos (Supplementary Fig. 6a, b). Whole-mount staining of LYVE1 indicated that anterior cardiac lymphatic vessels were reduced, whereas posterior lymphatics were comparable between control and Pofut $1^{\mathrm{cKO}}$ embryos (Supplementary Fig. 7). These results demonstrate that POFUT1 in the endocardial/coronary endothelial lineage primarily regulates the formation of coronary arteries and may be necessary for maintaining the arterial fate of coronary plexus.

POFUT1 regulates expansion of coronary angiogenic precursors. The hypoxia and VEGFA gradient in the inner myocardium suggest that this is the site undergoing vasculogenesis during coronary artery development. Since DLL4 is known to be highly expressed by vascular tip cells ${ }^{23}, 24$ during sprouting angiogenesis, an increased number of DLL4-positive coronary plexuses in the inner myocardium of Pofut $1^{\mathrm{cKO}}$ embryos would point to increased vasculogenesis following loss of Pofut1. We therefore examined the expression of VEGFR2 and VEGFR3, which are two major angiogenic markers. VEGFR2 was evenly expressed by all coronary endothelium, while VEGFR3 staining revealed two distinct coronary endothelial cell populations in the myocardium of E16.5 hearts (Supplementary Fig. 8a, b). One population expressing high levels of VEGFR3 (VEGFR3 ${ }^{\text {high }}$ ) in newly-formed coronary plexuses was located in the inner myocardium. The other population that expressed low levels of VEGFR3 (VEGFR3 ${ }^{\text {low }}$ ) was present in the outer myocardium and includes endothelial cells of mature coronary arteries (Fig. 3a, b, Supplementary Fig. 8b). In Pofut $1^{\mathrm{cKO}}$ hearts, the population of VEGFR $3^{\text {high }}$ cells was greatly expanded (Fig. 3a, b).

We have previously shown that ventricular endocardial cells labeled by $N f a t c 1$ are a major source of coronary arterial endothelium ${ }^{7}$. To determine whether these VEGFR3 ${ }^{\text {high }}$ cells are derived form endocardium, we performed co-immunostaining of VEGFR3 with $\mathrm{Nfatc1}^{\mathrm{Cre}}$ lineage marker GFP from E11.5 to E16.5. VEGFR3 was highly expressed in a subset of endocardial cells at the base of trabeculae, intimately contacting the compact myocardium at E11.5 when the earliest coronary plexus starts to form in the coronary sulcus and interventricular septum (Supplementary Fig. 9). These VEGFR3 ${ }^{\text {high }}$ endocardial cells clearly protruded into the myocardium and represented the earliest endocardial 'sprouts'. The VEGFR3 ${ }^{\text {high }}$ cell population expanded in the myocardium as a coronary plexus after E12.5. At E15.5 (soon after coronary circulation begins around E14.5), the fast growing compact myocardium received active perfusion from newly-established coronary arteries located in the outer layer, which was thus less hypoxic. In contrast, the inner layer distant from the coronary arteries was more hypoxic (Fig. 2e). These findings suggest the inner myocardium is the region continuously undergoing vasculogenesis after the initiation of coronary circulation. Consistently, endothelial cells located in the inner myocardium expressed high levels of VEGFR3, while the endothelial cells in mature arteries located in the outer myocardium, express low levels of VEGFR3 (Fig. 3a, Supplementary Fig. 9). Importantly, lineage tracing and quantification of VEGFR $3^{\text {high }}$ cells at E16.5 showed that the majority $(\sim 70 \%)$ of VEGFR 3 high cells were derived from $\mathrm{Nfatc1+}$ endocardial cells, and this proportion was increased in Pofut $1^{\text {cKO }}$ hearts (Supplementary Fig. 8c, d; Supplementary Fig. 9). To further determine the temporal change of VEGFR3 ${ }^{\text {high }}$ cells in the developing coronary arteries, we compared the VEGFR3 expression in the Nfact1+ coronary progeny (GFP labeled) between E13.5 and E16.5. The results showed that $>90 \%$ GFP-expressing cells expressed high levels of VEGFR3 at E13.5, while this number decreased to $60 \%$ at E16.5 (Supplementary Fig. 9). This temporal change was well correlated with VEGFR3 ${ }^{\text {high }}$ angiogenic precursors undergoing vasculogenesis in the forming plexus at E13.5, while reduced VEGFR3 expression was observed in the endothelial cells of mature coronary arteries at E16.5.

We further characterized these VEGFR3 $3^{\text {high }}$ cells by co-staining of VEGFR3 with angiogenic markers, including VEGFR2, pVEGFR2 and VEGFA. The results showed that VEGFR $3^{\text {high }}$ cells were a subpopulation of VEGFR2-expressing cells with high levels of pVEGFR2 and phosphorylated PAXILLIN (pPAXILLIN), which is involved in cell migration by regulating cell focal adhesion turnovers ${ }^{37,38}$ (Supplementary Fig. 10). VEGFA was also highly enriched in the inner myocardium where VEGFR $3{ }^{\text {high }}$ cells were located (Supplementary Fig. 10). These findings suggest that these VEGFR3 ${ }^{\text {high }}$ endothelial cells were actively migratory and angiogenic. Importantly, like VEGFR $3^{\text {high }}$ cells, the zone of pPAXILLINexpressing cells was expanded in Pofut $1^{\mathrm{cKO}}$ hearts (Fig. 3c). At high magnification, PECAM1-, DLL4- or VEGFR3-stained heart sections showed that within the inner myocardium of control hearts, $10 \%$ of PECAM1-positive cells had filopodia-like protrusions (microspikes) (Fig. $3 \mathrm{~d}-\mathrm{f})^{26,39}$, similar to protrusions seem in vascular tip cells. More PECAM1-positive endothelial cells possessed these protrusions in Pofut $1^{\mathrm{cKO}}$ hearts (Fig. 3d). Consistently, in vitro spheroid sprouting assays using HUVECs showed that knockdown of POFUT1 by shRNA resulted in excessive angiogenic sprouting (Fig. $3 g$ ). We next examined the proliferation by EdU incorporation, and found that VEGFR $3^{\text {high }}$ cells were more proliferative than the overall population of coronary endothelial cells expressing PECAM1, and Pofut1 deletion promoted proliferation of all endothelial cells (Fig. $3 \mathrm{~h}$, i). Together, these results suggest that the VEGFR3 ${ }^{\text {high }}$ cells serve as angiogenic precursor cells for coronary artery formation, and the angiogenic activity of these cells is negatively regulated by POFUT1.

Because hypoxia is known to promote coronary vascular formation through VEGFA/VEGFR2 signaling 7 , 40, the inner myocardium is more hypoxic and expresses more VEGFA than the outer myocardium during coronary artery development, and in Pofut $1^{\text {cKO }}$ hearts this hypoxic/VEGFA-expressing zone is greatly expanded (Fig. 2e-h), we examined expression of VEGFR2 and its activated phosphorylated form (pVEGFR2) by immunostaining and western blotting. The result indicated an increased number of VEGFR2-expressing cells and levels of pVEGFR2 in the inner myocardium of Pofut $1{ }^{\mathrm{cKO}}$ hearts (Fig. 3j, 1 and Supplementary Fig. 11). Furthermore, the phosphorylation of ERK1/2 (pERK1/2), a downstream target of VEGFR2, was elevated in the coronary angiogenic cells in the inner myocardium (Fig. 3k, 1 and Supplementary Fig. 11). Together, these findings suggest that the increased VEGFR2 signaling may contribute to the over-vasculogenesis phenotype in Pofut $1^{\mathrm{cKO}}$ hearts.

We validated the coronary phenotypes by deletion of Pofut1 in all vascular endothelium using Tie ${ }^{C r e}$ mice. As expected, the coronary phenotype was more severe, leading to embryonic death (Supplementary Table 1). Whole-mount PECAM1 staining indicated absence of the main coronary arteries in hearts of E16.5 pan-endothelial Pofut1 knockout embryos (Supplementary Fig. 12a). Immunostaining for IB4 and VEGFR3 showed markedly increased coronary plexuses (Supplementary Fig. 12b) and coronary angiogenic cells in the inner myocardium (Supplementary Fig. 12c). Further analysis using SMMHC 
a

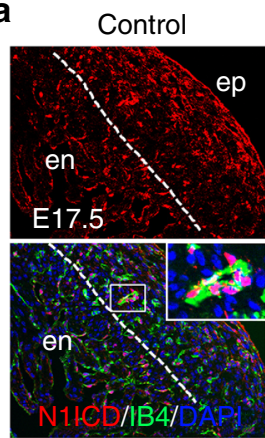

b

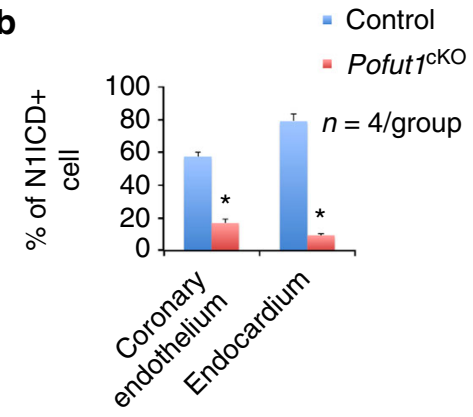

C
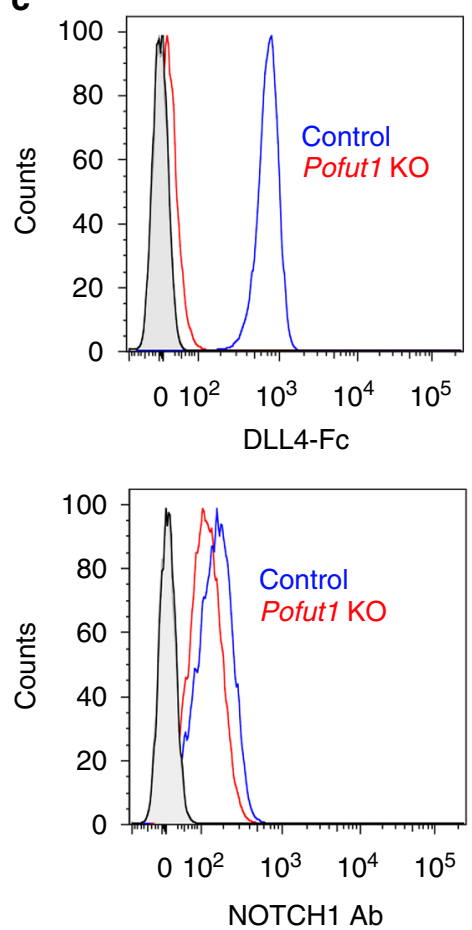

d
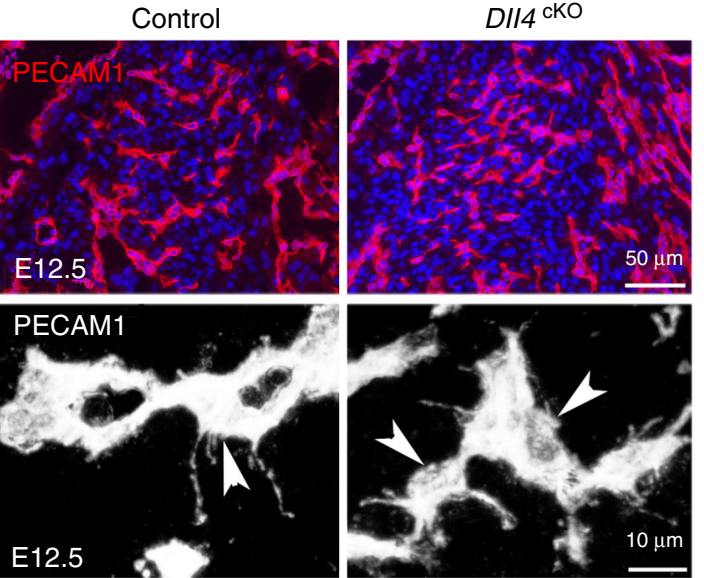

e
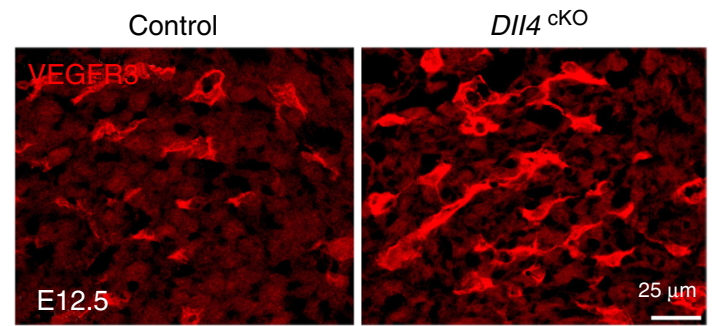

Control

- DII4 ${ }^{\mathrm{cKO}}$ $n=4 /$ group

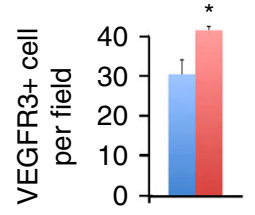

f
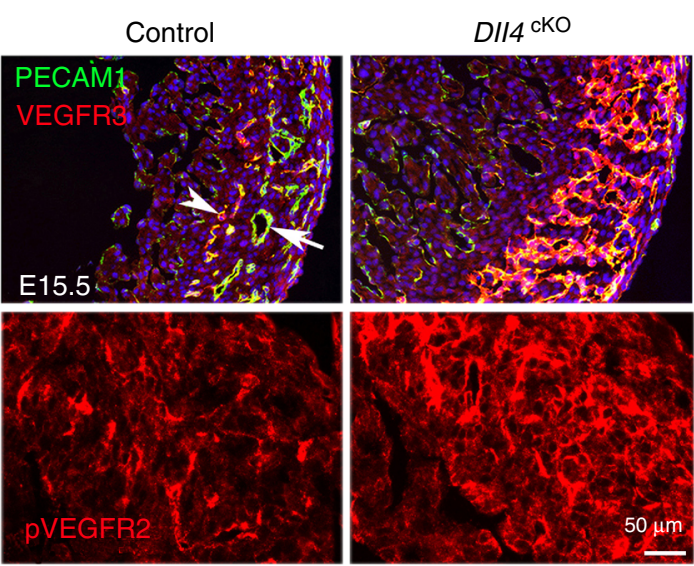

g
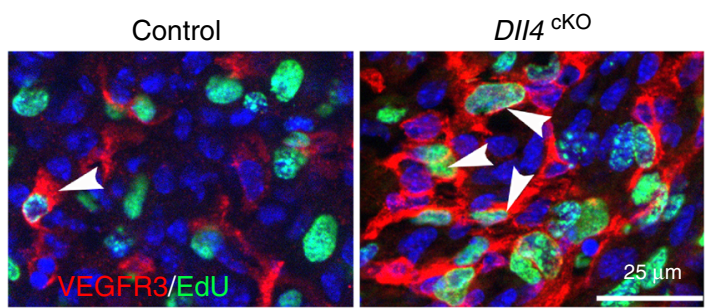

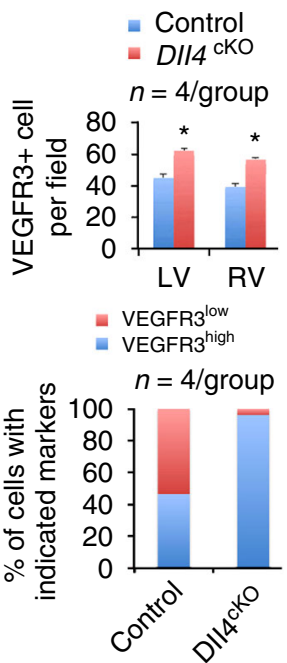

- Control

- DII4 cKO $n=3 /$ group

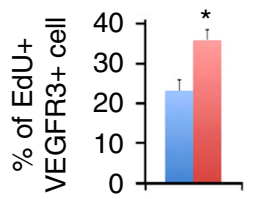

Fig. 4 POFUT1 regulates coronary artery formation via DLL4/NOTCH1 signaling. a, b Co-staining for N1ICD and IB4 showing reduced expression of N1ICD in coronary plexus of E17.5 Pofut ${ }^{\mathrm{CKO}}$ heart. Dotted line separates trabeculae from compact myocardium. c DLL4-Fc binding and NOTCH1 extracellular domain antibody binding to control (blue profiles) and Pofut1 knockout (Pofut1 KO) (red profiles) CHO cells showing reduced DLL4 binding by Pofut1 KO cells, which have normal NOTCH1 expression. Grey profiles reflect secondary antibody alone. d, e PECAM1 and VEGFR3 staining for coronary angiogenic cells (arrowhead) in coronary plexuses at E12.5, one-day after DII4 deletion, showing increased PECAM1+ area, the number of PECAM1+ tip-like or VEGFR3 + cells in DII4 $4^{\mathrm{cKO}}$ (DII4f/f; Cdh5 ${ }^{\text {CreERT2}}$ ). f PECAM1 and VEGFR3 staining for coronary angiogenic cells (arrowhead) and arteries (arrow) at E15.5, one-day after DII4 deletion, shows increased number of VEGFR3 ${ }^{\text {high }}$ cells in DII4CKO. ${ }^{*} p<0.001 . \mathbf{g}$ EdU labeling of VEGFR3+ coronary angiogenic cells at E15.5, 1 day after DIl4 deletion, shows increased proliferation of VEGFR3 cells (arrowheads). All bar charts represent mean \pm SD;

${ }^{*} p<0.001$. en/ep, endocardium/epicardium 
a
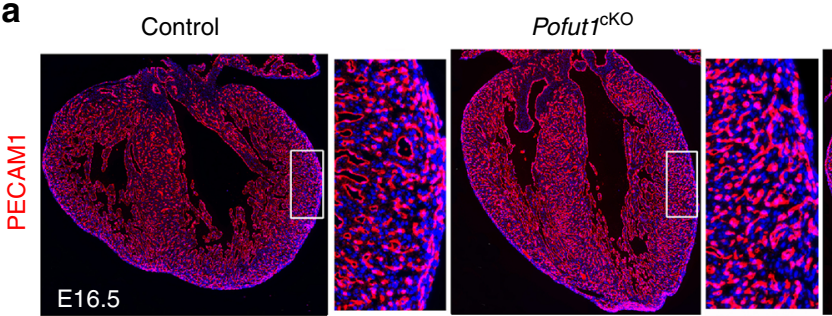

Pofut1; Vegfr3 ${ }^{\text {DKO }}$
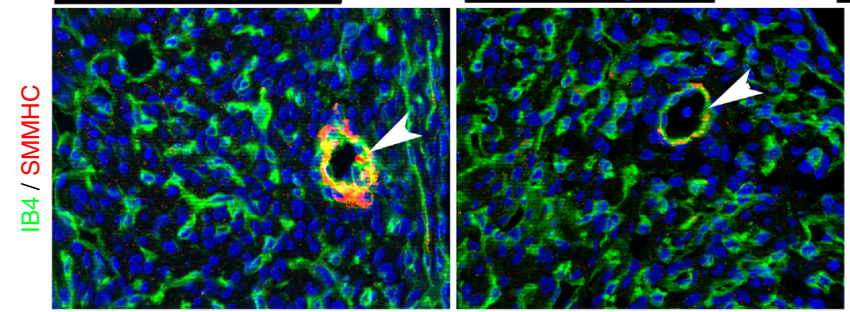

b

Control

$D I I 4^{\mathrm{K} O}$
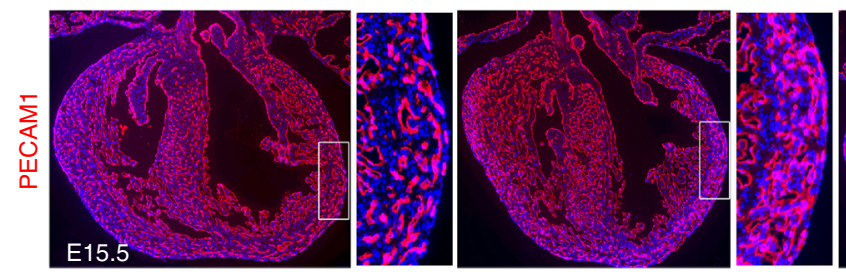

DII4; Vegfr3 ${ }^{\mathrm{DKO}}$
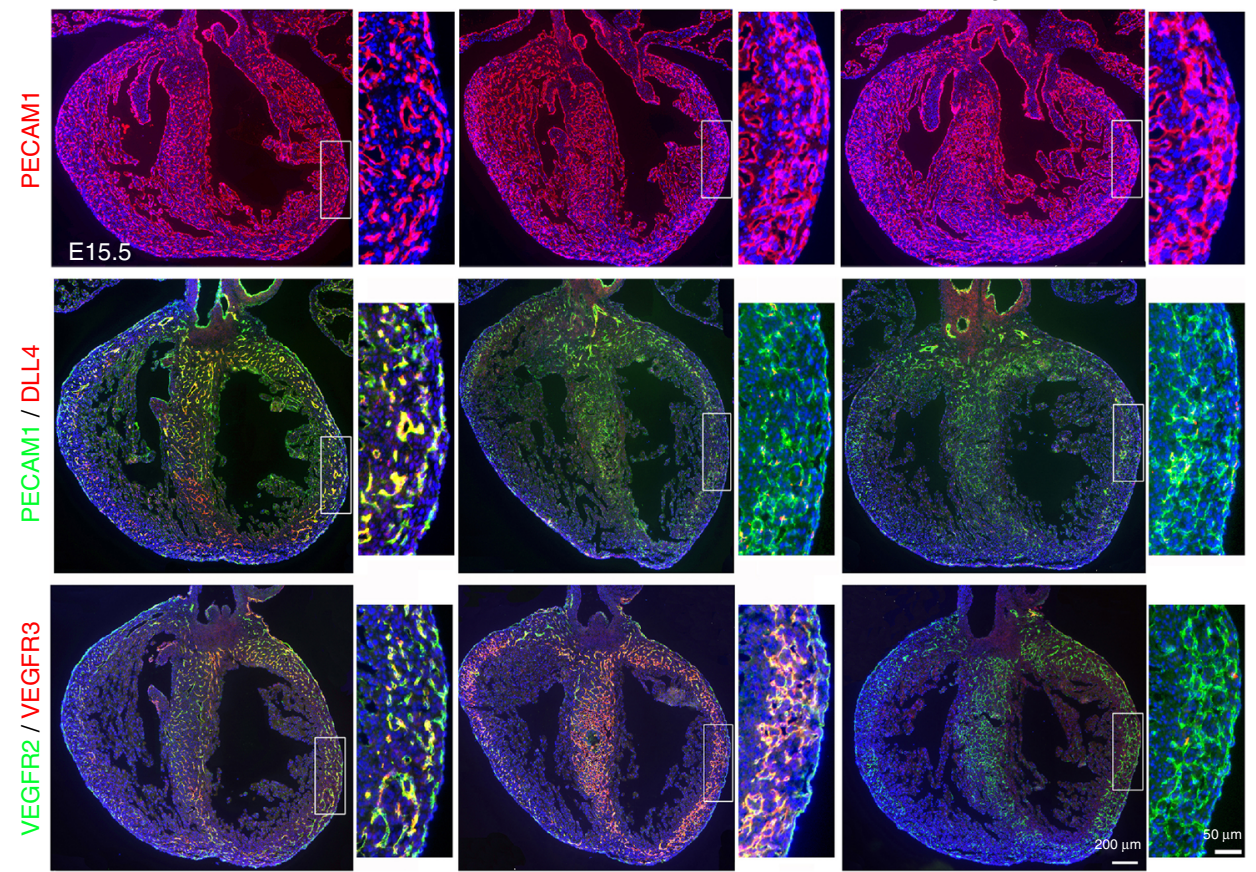

Fig. 5 Deletion of Vegfr3 fails to rescue coronary defects in Pofut $7^{\mathrm{KKO}}$ or DII4 ${ }^{\mathrm{cKO}}$ mice. a PECAM1 staining and IB4/SMMHC co-staining shows increased coronaries in E16.5 heart with endocardial Pofut1 deletion, which cannot be rescued by simultaneous deletion of Vegfr3. IB4 and SMMHC co-staining shows defective major coronary arteries (arrowhead) remain in the double mutants. b PECAM1 staining shows increased coronaries in E15.5 DII4cKO heart by induced DII4 deletion at E14.5, which cannot be rescued by simultaneous deletion of Vegfr3. PECAM1/DLL4 or VEGFR2/VEGFR3 co-staining shows effective deletion of Dll4 or Vegfr3

antibodies confirmed no major coronary arteries in these knockout hearts (Supplementary Fig. 12d). Co-immunostaining for PECAM1 with SM $22 \alpha$ or $\alpha$ SMA confirmed greatly increased small coronary vessels that were unable to mature into larger arteries (Supplementary Fig. 12e). Consistent with the endocardial deletion of Pofut1, the pan-endothelial deletion resulted in significantly increased proliferation of coronary endothelial cells (Supplementary Fig. 12f, g). Altogether, these observations demonstrate that POFUT1 controls coronary artery development by regulating angiogenic functions and proliferation of coronary precursor cells, which express high levels of VEGFR3.

POFUT1 controls NOTCH signaling in coronary artery formation. To investigate $\mathrm{NOTCH}$ signaling downstream of POFUT1, we examined expression of NOTCH1, N1ICD, and
DLL4 in fetal hearts. Endocardial deletion of Pofut1 did not appear to affect levels of membrane NOTCH1 or DLL4 in individual cells at E14.5 (Supplementary Fig. 13), yet resulted in markedly decreased expression of nuclear N1ICD in endocardium and coronary arterial endothelium at E14.5 and E17.5 (Fig. 4a, b and Supplementary Fig. 13). Ligand binding to CHO cells lacking POFUT1 had essentially no binding of soluble DLL4-Fc ligand but equivalent expression of NOTCH1 at the cell surface, indicated by binding of antibodies against NOTCH1 extracellular domain (Fig. 4c and Supplementary Table 2). We therefore deleted Dll4 in endocardial lineage by crossing floxed Dll4 mice with $\mathrm{Nfatc1}^{\mathrm{Cre}}$ mice to determine if loss of Dll4 would mimic loss of Pofut1 in coronary artery development. However, the deletion was embryonic lethal before E11.5 and the null embryos were already runted around E10.5 (Supplementary 
a

- Pofut ${ }^{\mathrm{ckO}}(n=31)$

- Pofut $1 ;$ Vegfr2 $^{\mathrm{DKO}}(n=15)$

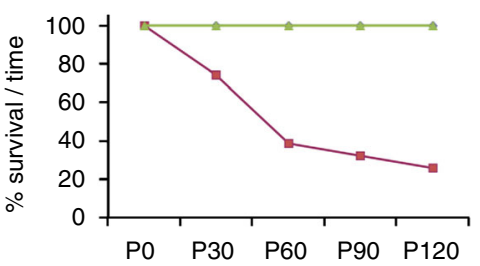

b

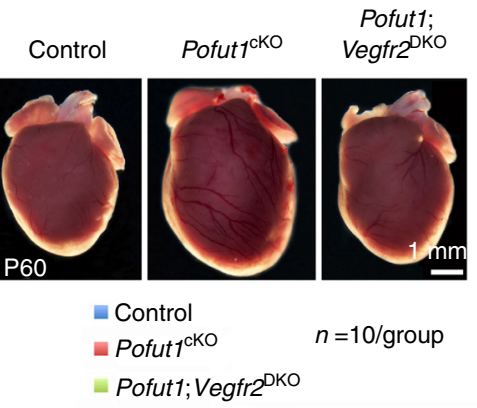

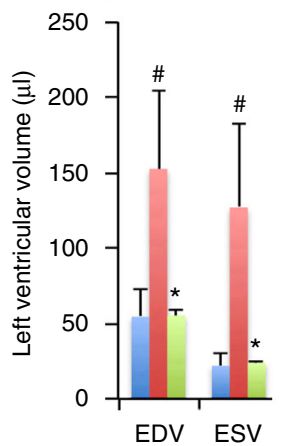

d

d Control
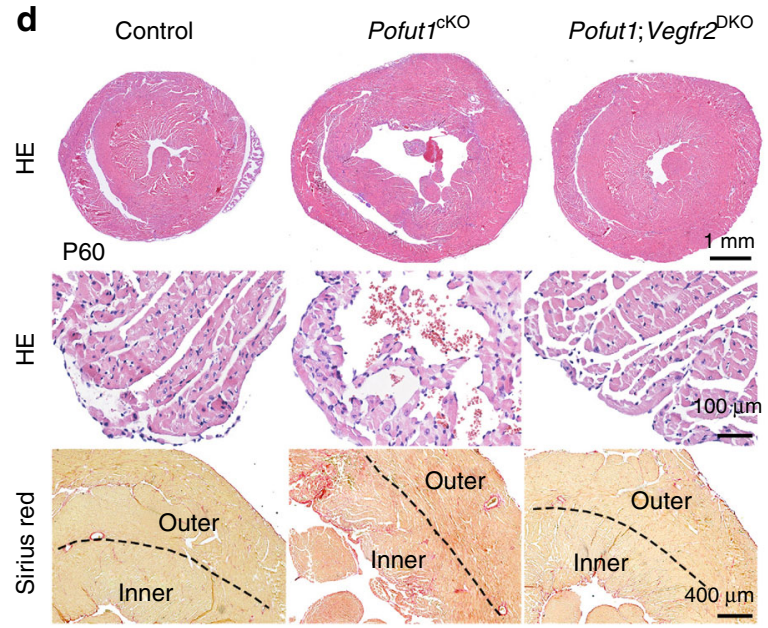

f
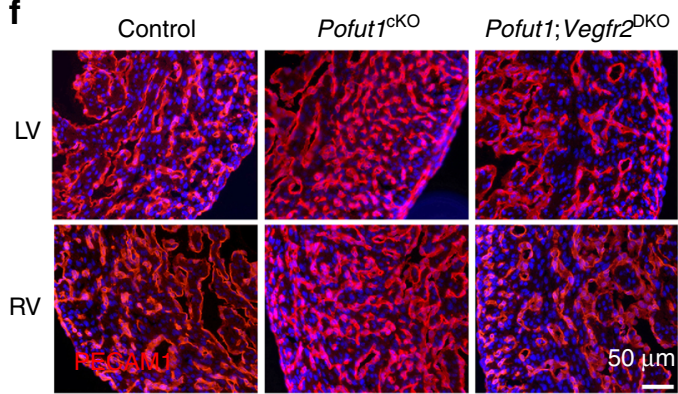

h
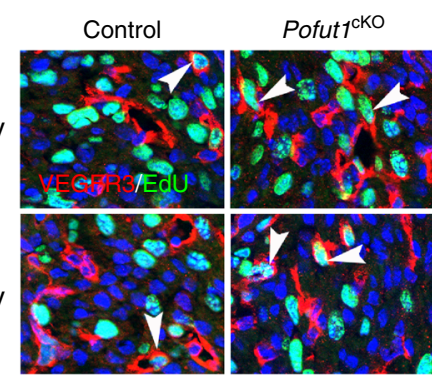

Pofut $1 ;$ Vegfr2 ${ }^{\mathrm{DKO}}$
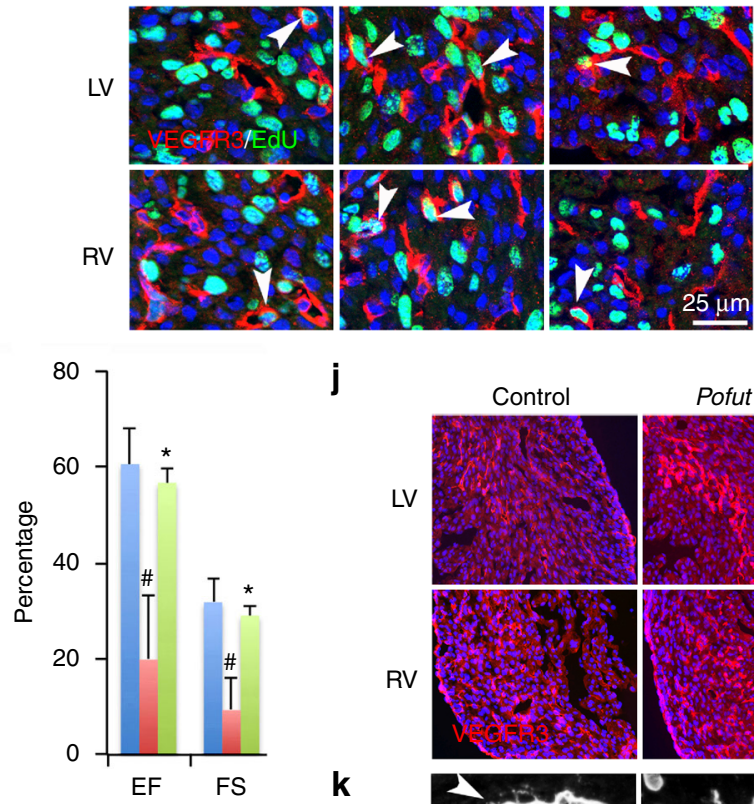

k

Pofut1; Vegfr2 ${ }^{\mathrm{DKO}}$

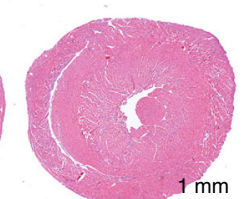

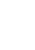
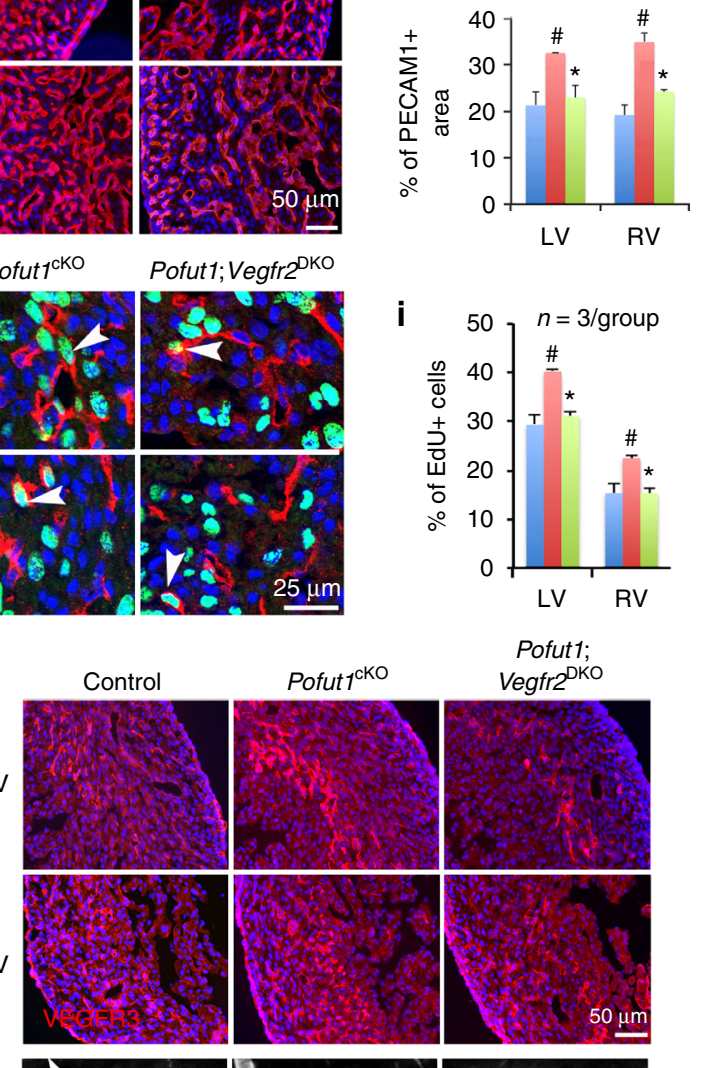

LV
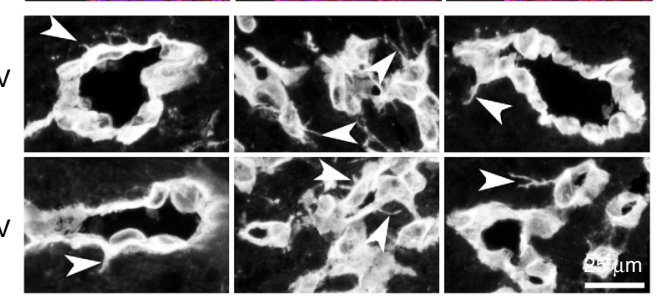

e
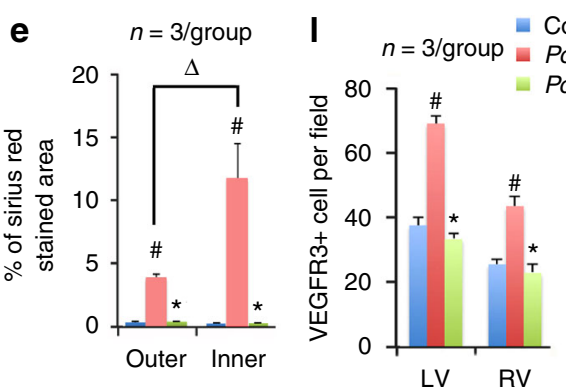

Control

Pofut $1 ;$ Vegfr2 ${ }^{\mathrm{DKO}}$

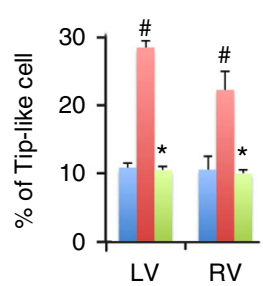

Fig. 6 VEGFR2 mediates POFUT1 function for coronary artery formation. a Survival curve of Pofut $7^{c K O}$ and Pofut1; Vegfr2 ${ }^{D K O}$ mice. b Gross view of P60 hearts. c Heart/body ratio of P60 mice. \#p $<0.01$ between control and Pofut ${ }^{\mathrm{CKO}}{ }^{*}{ }^{\star} p<0.01$ between Pofut ${ }^{\mathrm{CKO}}$ and Pofut1; Vegfr $2^{\mathrm{DKO}}$. Echocardiography shows that cardiac function of 2-month Pofut1; Vegfr2 ${ }^{D K O}$ mice are restored. \#, ${ }^{*} p<0.001$. EDV/ESV, end diastolic/end systolic volumes; EF/FS, ejection fraction/fractional shortening. d Cardiac histology shows no sign of myocardial infarction in P60 Pofut7; Vegfr2 ${ }^{D K O}$ hearts. e Quantification of Sirius Red staining. $\# p<0.01$ between control and Pofut $7{ }^{\mathrm{KKO}},{ }^{\star} p<0.01$ between Pofut $7 \mathrm{CKO}^{\mathrm{K} O}$ and Pofut7; Vegfr $2^{\mathrm{DKO}} . \Delta p<0.01$ between outer and inner.

f, $\mathbf{g}$ PECAM1 staining shows restored coronary network in E16.5 Pofut1; Vegfr2 ${ }^{\text {DKO }}$ hearts. $\mathbf{h}$, i VEGFR3/EdU co-staining shows reduced proliferation of angiogenic cells (arrowheads) to normal levels in P60 Pofut1; Vegfr2 ${ }^{\mathrm{DKO}}$ hearts. \#, ${ }^{\star} p<0.05$. j, I VEGFR3 staining shows a restored number and distribution of angiogenic cells in E16.5 Pofut1; Vegfr2 ${ }^{\mathrm{DKO}}$ hearts. Mean \pm SD; \#, ${ }^{\star} p<0.001$. k, I PECAM1 staining shows a restored number of tip-like cells (arrowheads) in E16.5 Pofut1; Vegfr2 ${ }^{\mathrm{DKO}}$ hearts. Mean $\pm \mathrm{SD} ; \#,{ }^{*} p<0.001$ 


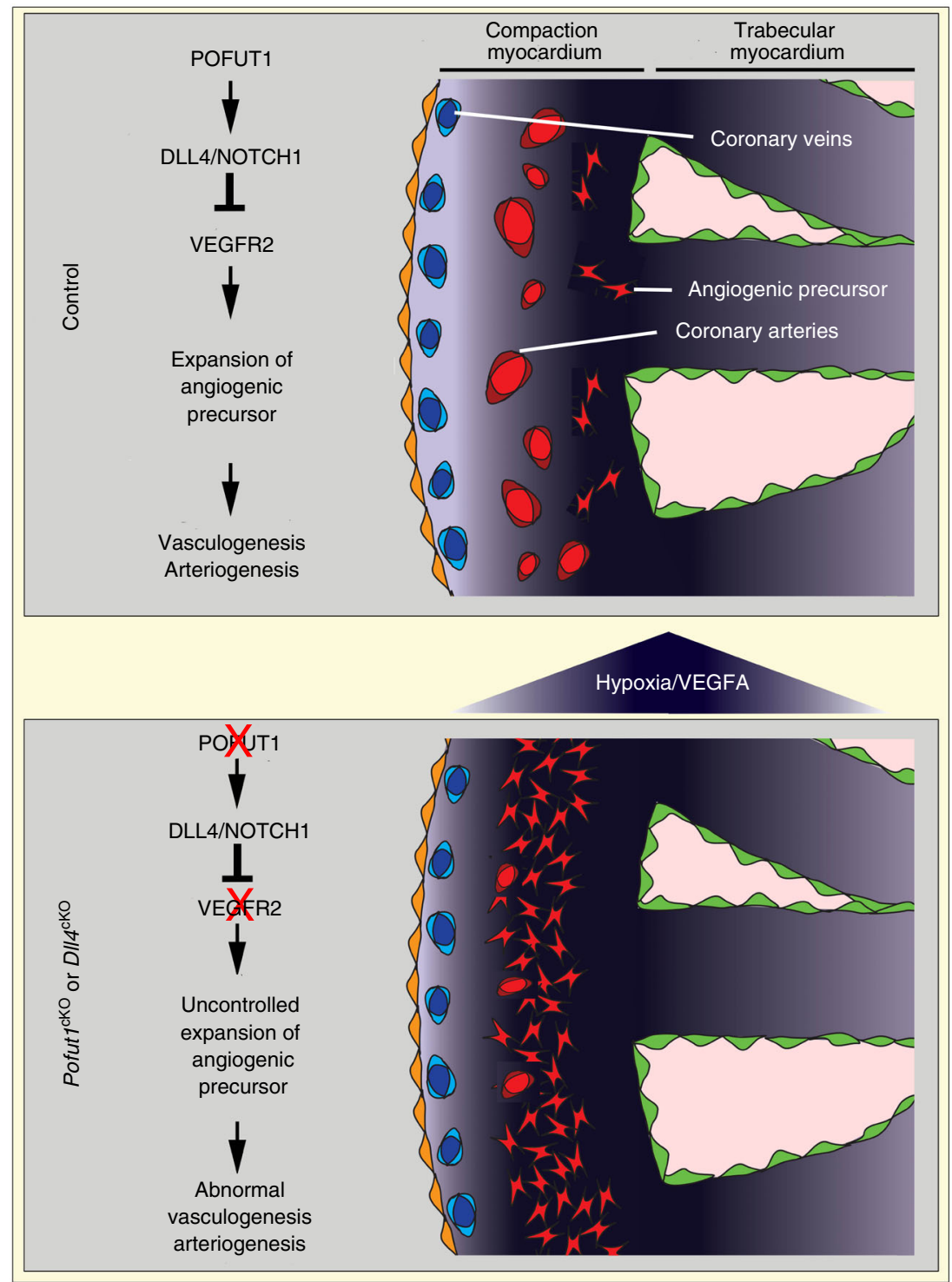

Fig. 7 POFUT1 regulates proliferation and angiogenic functions of precursor cells for coronary artery development. During coronary artery development, coronary angiogenic precursor cells give rise to coronary arterial plexuses in the hypoxic and VEGFA-rich inner myocardium by vasculogenesis. These plexuses then invade and fuse to form coronary arteries in the outer myocardium through arteriogenesis. Proliferation of coronary angiogenic cells during coronary vasculogenesis is modulated by POFUT1/DLL4/NOTCH1/VEGFA/VEGFR2 signaling axis. Deletion of Pofut1 suppresses DLL4/NOTCH1 signaling, which in turn elevates VEGFA/VEGFR2 signaling. Augmented VEGFA/VEGFR2 signaling promotes proliferation and angiogenic functions of coronary precursor cells, resulting in excessive non-functional coronary plexuses, which are unable to mature into coronary arteries

Fig. 14). To overcome the early lethality, we used the inducible endothelial Cre line Cdh5(PAC)/CreERT2 ${ }^{41}$ to delete Dll4 at E11.5 or E14.5. PECAM1 and VEGFR3 co-immunostaining showed that deletion at either time point resulted in increased formation of coronary angiogenic cells and coronary plexuses (Fig. 4d-f). Of note, the deletion of Dll4 at E14.5 appeared to promote VEGFR3 ${ }^{\text {Low }}$ endothelial cells in the outer myocardial wall to become VEGFR3 ${ }^{\text {high }}$ angiogenic cells (Fig. 4f, Supplementary Fig. 15). In addition, loss of Dll4 decreased NOTCH signaling and augmented pVEGFR2 and the proliferation of VEGFR $3^{\text {high }}$ cells (Fig. 4f, g, Supplementary Fig. 16), similar to lack of DLL4 binding to NOTCH1 due to deletion of Pofut1.

NOTCH inhibition by the gamma-secretase inhibitor DAPT may increase microtubule dynamics ${ }^{42}$, and increased microtubule dynamics is correlated with increased cell migration ${ }^{43}$. We therefore sought to determine whether inhibition of $\mathrm{NOTCH}$ signaling would promote VEGFA-induced cell migration in cultured HUVEC cells. The results of time-lapse imaging showed that either DAPT treatment or shRNA knockdown of POFUT1 or DLL4 significantly increased cell mobility indicated by increased velocity and mean square displacement (Supplementary Fig. 17a-d). Co-staining for acetylated and total microtubules showed that inhibition of NOTCH signaling decreased the ratio of acetylated to total microtubules (Supplementary Fig. 17e, f), indicating increased microtubule plasticity as acetylation of microtubules is associated with more stable, long lived and less dynamic microtubules ${ }^{44}$. The increased cell mobility was correlated with an increased number of filopodia (Supplementary Fig. $17 \mathrm{~g}, \mathrm{~h}$ ). These findings support the regulation of coronary angiogenic cell migratory behavior by POFUT1 through regulating DLL4/NOTCH1-induced signaling in coronary artery formation. 
VEGFR2 mediates POFUT1 function in coronary artery formation. Since Pofut1 or Dll4 deletion promoted VEGFR3 expression and increased VEGFR2 phosphorylation, we next investigated whether signal alterations through VEGFR3 and VEGFR2 are responsible for the coronary phenotype in Pofut $1^{\mathrm{cKO}}$ or Dll4 ${ }^{\mathrm{cKO}}$ embryos. We first investigated the role of $V e g f r 3$ and Vegfr2 in coronary artery development by deleting either gene using the $N f a t c 1^{C r e}$ driver. The results showed that VEGFR3 suppresses and VEGFR2 promotes coronary vasculogenesis (Supplementary Fig. 18a-c). To directly test whether VEGFR3 and VEGFR2 work downstream of NOTCH signaling in coronary artery development, we simultaneously deleted Vegfr3 and Dll4, or Vegfr3 and Pofut1. Consistently, inactivation of $V e g f r 3$ did not alleviate the coronary phenotype resulting from Dll4 or Pofut1 deletion (Fig. 5a, b, Supplementary Fig. 18d, e). Strikingly, however, inactivation of VEGFR2 fully rescued the lethality of Pofut ${ }^{\text {cKO }}$ mice (Fig. 6a). Compared to their littermates with deletion of Pofut1, mice with double deletion of Pofut1 and Vegfr2 (Pofut1;Vegfr $2^{\mathrm{DKO}}$ ) had normal heart size (Fig. 6b, c) and cardiac function, including end-diastolic and end-systolic volumes, as well as ejection fraction and fraction shortening (Fig. 6c). Histologically, Pofut1; Vegfr $2^{\mathrm{DKO}}$ mice had no sign of myocardial infarction by HE staining, nor was there increased cardiac fibrosis by Sirius Red staining (Fig. 6d, e). Immunostaining for PECAM1 revealed that Pofut 1 ;Vegfr $2^{\mathrm{DKO}}$ embryos had coronary vasculature that was comparable to controls (Fig. 6f, g). Co-immunostaining for VEGFR3 and EdU indicated that the coronary vasculature in Pofut 1 ;Vegfr $2^{\mathrm{DKO}}$ embryos contained angiogenic cells with a normal proliferative rate (Fig. 6h, i). Consistently, Pofut1; $V e g f r 2^{\text {DKO }}$ embryos had a normal number of VEGFR $3^{\text {high }}$ angiogenic precursor cells (Fig. 6j-1). These observations indicate that augmented VEGFR2 signaling in coronary angiogenic precursors of Pofut $1^{\mathrm{cKO}}$ mice likely drives the overall coronary phenotype, including increased proliferation of coronary angiogenic precursors and formation of coronary plexuses, with defective arteriogenesis.

\section{Discussion}

Collectively, our studies suggest that VEGFR3 ${ }^{\text {high }}$ coronary angiogenic precursors undergo vasculogenesis to form coronary plexuses, which subsequently mature into large coronary arteries by arteriogenesis (Fig. 7). In this sequential process, POFUT1 regulates the proliferation of coronary angiogenic precursors and their functions through regulating the DLL4/NOTCH1/VEGFA/ VEGFR2 signaling axis. Disruption of Pofut1, which controls the strength of NOTCH ligand binding, or Dll4, results in coronary artery anomalies that lead to early-onset ischemic heart disease. We identify the primary early developmental defect as increased angiogenic cell proliferation and function, which promotes coronary vasculogenesis, but impairs subsequent arteriogenesis. The latter maturation defect is supported by defective recruitment of smooth muscle cells during arteriogenesis ${ }^{28-30,45}$. The defective coronary arteries fail to provide sufficient perfusion to meet the demands of the growing heart and cardiac functional output, resulting in severe myocardial hypoxia, and inevitably myocardial infarction and heart failure. In some cases, rupture of large coronary aneurysms causes large myocardial infarctions and early death of young mice.

POFUT1 modifies NOTCH receptors by transferring O-fucose to their epidermal growth factor (EGF)-like repeats ${ }^{46}$. This post-translational modification is essential for proper $\mathrm{NOTCH}$ ligand-receptor interactions and subsequent signaling $33-35,47$. Notch signaling is well known to inhibit sprouting angiogenesis during retinal and tumor angiogenesis ${ }^{23}, 24,48,49$. FRINGE, an enzyme that also modifies $\mathrm{NOTCH}$ receptors after POFUT1 modification, leads to preferential DLL4 binding to repress retinal angiogenesis $^{25}$. However, whether POFTU1 is required for angiogenesis or vascular development has not yet been determined. This study for the first time documents that POFUT1 is required for coronary artery formation through regulating vasculogenesis and arteriogenesis. Like FRINGE, POFUT1 promotes NOTCH signaling through DLL4 binding ${ }^{25}$.

We have previously shown that endocardium labeled by $\mathrm{Nfatc1}$ has a major contribution to coronary arterial endothelium ${ }^{7}$. Deletion of Pofut1 in $\mathrm{Nfatcl}^{+}$lineage severely impairs coronary artery development and has minor effects on coronary vein or lymphatic vessel development. To better explain the cell type-specific phenotype caused by Pofut1 deletion, the contribution of the $\mathrm{Nfatcl}^{+}$lineage to different coronary vessels was analyzed by co-immunostaining of cell type-specific markers with the $\mathrm{Natcl}^{+}$lineage reporter, and the experiments were carried out independently in Zhou and Harvey laboratories. The results confirm that the $\mathrm{Nfatcl}^{+}$endocardial lineage has a major contribution to embryonic coronary arteries $(>70 \%)$, a minor contribution to coronary vein $(<50 \%)$, and no, or very little, contribution to cardiac lymphatic endothelium (Supplementary Figs. 19-21).

Our study has identified two distinct endothelial cell subpopulations in developing coronary arteries. They are VEGFR3 $3^{\text {low }}$ cells in the mature coronary arteries in the outer myocardium, and VEGFR $3^{\text {high }}$ cells in the primitive coronary plexus within the hypoxic inner myocardium where VEGFA is enriched. These VEGFR $3^{\text {high }}$ cells also express high levels of DLL4. Both VEGFR3 and DLL4 are highly expressed in vascular tip cells of developing vessels in the retina and angiogenic tumor vessels ${ }^{50}$. Furthermore, VEGFR $3^{\text {high }}$ cells are a subpopulation of VEGFR2-expressing cells with high levels of pVEGFR2, suggesting that they are actively angiogenic. PAXILLIN is a downstream target of VEGFA signaling and regulates endothelial cell migration by modulating focal adhesion turnover ${ }^{37,38}$. Our results show that VEGFR3 high and VEGFR $3^{\text {low }}$ cells have high and low levels of pPAXILLIN, respectively, suggesting that VEGFR $3^{\text {high }}$ cells are more mobile. In addition, co-immunostaining of VEGFR3 with the $\mathrm{Nfatcl}^{\mathrm{Cre}}$ mediated GFP lineage reporter reveals that VEGFR $3^{\text {high }}$ cells are derived from the endocardium. Together, our findings suggest that the VEGFR $3^{\text {high }}$ cells arise from the endocardium and serve as an angiogenic precursor cell population for coronary artery development. Further, their angiogenic feature is controlled by POFUT1 via NOTCH signaling.

Angiogenic precursor cells form vascular plexuses through vasculogenesis and this process is regulated by $\mathrm{NOTCH}$ and VEGFA signaling $23,25,51$. Our study indicates that during formation of embryonic coronary arteries, VEGFR $3^{\text {high }}$ cells may represent a progenitor cell pool for continuous coronary artery formation to meet the increasing demands of the growing myocardium. Our model predicts that in normal coronary artery formation, VEGFR $3^{\text {high }}$ cells form plexuses in the inner myocardium and then organize into mature vessels under the regulation of a hypoxia-driven VEGFA gradient via VEGFR2. DLL4/ NOTCH1 interactions among the VEGFR $3^{\text {high }}$ cells modulate their proliferation, migration and maturation by suppressing VEGFA/VEGFR2 signaling. With depressed DLL4/NOTCH1 interactions caused by Pofut1 inactivation, VEGFA/VEGFR2 signaling intensifies in the VEGFR $3^{\text {high }}$ angiogenic cells, leading to expansion of this progenitor pool by increased proliferation, as well as an increase in angiogenic migration, possibly driven by an increased hypoxic stimulus. However, the excessive coronary angiogenic cells only form immature, non-functional plexuses. This promotes further increased hypoxia in the ventricular myocardium leading to 
increased VEGFA activity that amplifies the already dysregulated vasculogenesis by inducing the formation of more immature VEGFR $3^{\text {high }}$ angiogenic cells.

Deletion of Pofut1 or Dll4 also results in defective coronary arteriogenesis. JAG1/NOTCH3 signaling is known to promote smooth muscle cell recruitment and differentiation during vascular maturation $28-30,45$ and both genes are significantly downregulated by deletion of Pofut1. However, our rescue experiment would favor the notion that defective arteriogenesis is secondary to the early vasculogenesis defect, since disruption of Vegfr2 specifically in endocardium fully rescues both vasculogenesis and arteriogenesis defects in Pofut ${ }^{c K O}$ mice. Additionally, deletion of either Pofut1 or Dll4 results in expansion of coronary angiogenic cells, which are unable to become mature endothelial cells. Therefore, we suggest that disruption of the DLL4/NOTCH1/ VEGFA/VEGFR2 signaling axis by deletion of Vegfr2 rescues arteriogenesis defects in Pofut ${ }^{c K O}$ mice by repressing the number of angiogenic cells formed and allowing their conversion to mature vessels.

A potential redundant function between VEGFR2 and VEGFR3 in driving angiogenesis downstream of NOTCH signaling has been noted previously. VEGFR3 stimulation augments VEGFA-induced angiogenesis in the presence of VEGFR2 inhibitors, whereas antibodies against both VEGFR2 and VEGFR3 additively inhibit angiogenesis ${ }^{50}$. Similarly, VEGFR3 upregulation resulting from NOTCH signaling inhibition is able to drive retina angiogenesis without VEGF-VEGFR2 signaling ${ }^{52}$. Clinically, the inhibitors of VEGFA/VEGFR2 signaling show initial efficacy in treatment of tumors, but without lasting effects, which is thought to be due to a compensation from VEGFA/ VEGFR3 signaling ${ }^{53}$. However, our data do not support such compensation in the developing coronary arteries. Rather, they indicate that DLL4/NOTCH1/VEGFA/VEGFR2 is an essential molecular signaling axis underlying coronary artery development. Further investigation is needed to identify the non-redundant roles of VEGFR2 and VEGFR3 in the process, since they may function in a context-dependent manner ${ }^{46}, 50,52-54$.

The new mouse model of coronary artery anomalies and early-onset ischemic heart disease we describe herein will provide novel opportunities for gaining deeper insights into the developmental origins and genetic mechanisms of coronary vessel development. The information may have clinical implications in developing genetic screening methods for coronary artery anomalies and providing a developmental paradigm for regenerative medicine for coronary artery disease.

\section{Methods}

Mouse strains. Mouse housing and experiments were performed according to the protocols approved by the Institutional Animal Care and Use Committee of Albert Einstein College of Medicine. The tissue specific Pofut1 knockout mice were generated by crossing floxed Pofut 1 mice ${ }^{35}$ with Tnnt ${ }^{\text {Cre }}$ for cardiomyocytes ${ }^{55}$, Tbx $18^{\text {Cre }}$ for epicardium ${ }^{56}, \mathrm{Sm} 22 \alpha^{\mathrm{Cre}}$ for smooth muscle cells ${ }^{57}$, Mef2c Cre for secondary heart field ${ }^{58}$, Tiel ${ }^{\mathrm{Cre}}$ for pan-endothelial cells ${ }^{59}$ and $\mathrm{Nfatcl}^{\mathrm{Cre}}$ for endocardial lineage ${ }^{7}$. Similarly, endocardial lineage Dll4 knockout mice were generated by crossing floxed Dll4 mice ${ }^{60}$ with $\mathrm{Nfatcl}^{\mathrm{Cre}}$ mice. For inducible deletion of Dll4 in endothelial cells, inducible Cre line Cdh5(PAC)-CreERT2 ${ }^{41}$ was crossed with floxed Dll4 mice. To induce the deletion, tamoxifen was injected into pregnant female mice through IP at a specified time point, and the coronary arteries were analyzed at indicated time points. Endocardial lineage Vegfr 2 or Vegfr 3 knockout mice were generated by crossing floxed Vegfr 2 or Vegfr $3^{61}$ mice with $\mathrm{Nfatcl}^{\mathrm{Cre}}$ mice, respectively. Pofut1 and Vegfr2 endocardial lineage double knockout mice were generated by crossing floxed Pofut1 and floxed Vegfr2 mice with $\mathrm{Nfatcl}^{\mathrm{Cre}}$ mice. Pofut1 and Vegfr 3 endocardial lineage double knockout mice were generated by crossing floxed Pofut1 and floxed Vegfr3 mice with $\mathrm{Nfatcl}^{\mathrm{Cre}}$ mice. Inducible Dll4 and Vegfr3 endothelial double-knockout mice were generated by crossing floxed Dll4 and floxed Vegfr3 mice with Cdh5(PAC)-CreERT2 mice. Floxed Pofut 1 mice were maintained in mixed background, while all other mouse stains were in C57B6 background. Embryos were isolated and inspected according to expected developmental ages. Underdeveloped embryos were excluded from
} studies. Age matched adult mice and embryos were randomly selected during experiments. Noontime on the day of detecting vaginal plugs was designated as E0.5. The yolk sac or tail was used for PCR genotyping.

Measurement of cardiac function by echocardiography. Echocardiography was performed at the Einstein Cardiovascular Physiology and Imaging Core Facility to evaluate the cardiac functions of control, Pofut $1^{\mathrm{cKO}}$, and Pofut 1 ; Vegfr $2^{\mathrm{DKO}}$ mice of both sexes at two-month old. Investigators were blinded to genotypes of mice and subsequent data analysis. Mice were anesthetized by inhalation of $2 \%$ isoflurane in glass chamber and 1 to $1.5 \%$ isoflurane via a nose cone. Echocardiographic assessment was performed using a Vevo 770 ultrasound with a real time micro-visualization transducer applied parasternally to the shaved chest wall. Vevo 770 quantification software was used to measure left ventricular end-diastolic volume (LVEDV), left ventricular end-systolic volume (LVESV), the ejection fraction (EF) and fractional shortening (FS). Statistical significant comparisons among groups were performed by one-way analysis of variance following Turkey's post-test. $p<0.05$ was considered significance.

Micro-computed tomography (MicroCT). MicroCT was performed to access the coronary artery in P20 control and Pofut $1^{\mathrm{CKO}}$ mice as described previously ${ }^{62}$ Investigators were blinded to genotypes of mice and subsequent data analysis. In brief, the freshly euthanized mice were injected with bismuth contrast solution into descending aorta before the heart stops beating. Mice were immediately chilled in ice and immersion fixed in $2 \%$ paraformaldehyde (PFA) overnight. 2D microCT scans were acquired with a GE eXplore Micro-CT System (GE Healthcare) using a 400 cone beam filtered back projection algorithm, set to an $8-27-\mu \mathrm{m}$ micron slice thickness. Data were acquired in an axial mode, covering a volume of $2.0 \mathrm{~cm}$ in the $\mathrm{z}$ direction with a $1.04-\mathrm{cm}$ field of view. During post-processing, a 40,000 gray-scale value was set as a threshold to eliminate noise with minimal sacrifice of vessel visualization. The micro-CT data were processed using real-time $3 \mathrm{D}$ volume rendering software and Microview software to reconstruct three 2D maximumintensity projection images ( $x, y, z$ axes) from the raw data. Quantification was performed using a modified Image ProPlus 5.0 algorithm (Media Cybernatics). Data are expressed as vessel number, representing total number of vessels, of specified diameter counted in 350-400 z sections.

Histology and immunostaining. The preparation of paraffin and frozen sections and immunostaining were carried out following standard protocol as described previously ${ }^{63}$. Briefly, for paraffin sections, embryonic or adult hearts were freshly isolated in PBS, fixed in $4 \%$ PFA at $4{ }^{\circ} \mathrm{C}$ overnight, washed in PBS, dehydrated through a serial of gradient ethanol, cleared in xylene and embedded in paraffin. The hearts were then cut at $6 \mu \mathrm{m}$ and the tissue sections were mounted on positive charged slides. HE and Sirius red staining were performed using the standard protocol to evaluate the histology and pathology of the heart. For frozen sections, the hearts were isolated freshly, fixed in $4 \%$ PFA at $4{ }^{\circ} \mathrm{C}$ for a few hours dependent on the size of the tissues, soak in $15 \%$ and $30 \%$ sucrose sequentially and embedded in OCT compounds with orientation for front sections. The hearts were then cut at $8 \mu \mathrm{m}$, mounted on positive charged slides, post-fixed in cold ethanol and acetone (1:1) solution for $5 \mathrm{~min}$ and stored at $-80^{\circ} \mathrm{C}$. Tissue sections were air dried for 45 min before staining. Tissue sections were blocked with $5 \%$ normal horse or goat serum for $1 \mathrm{~h}$ at room temperature (RT) and incubated with primary antibodies with indicated dilution in blocking buffer at $4{ }^{\circ} \mathrm{C}$ overnight. Then the tissues were incubated with secondary antibodies conjugated with the Alexa 568 or 488 fluorescence dyes for $1 \mathrm{~h}$ at RT. To visualize the tip-like cell morphology (the microspikes or filopodia-like protrusion structure), the tyramide signal amplification system (PerkinElmer) was used for PECAM1 antibody staining. Antibody information was listed in Supplementary Table 3. Images were taken by a Zeiss Observer Z1 or Leica SP5 confocal microscope. All imaging quantifications were carried out blindly using Image J.

Detection of myocardial hypoxia. Hypoxia in embryonic or postnatal hearts was detected using Hypoxyprobe Kit (Hypoxyprobe Inc). The pregnant female mice or postnatal mice were injected with Hypoxyprobe ${ }^{\text {TM }}-1$ through intraperitoneal (IP) at a concentration of $60 \mathrm{mg} / \mathrm{kg}$ body weight After $90 \mathrm{~min}$ pulse, the hearts were isolated and processed for frozen section as described above. Then the hypoxia was detected by immunostaining of Hypoxyprobe antibody and Alexa Fluor 488 goat anti-mouse IgG secondary antibody. The stained tissues were photographed using a Zeiss Observer Z1 microscope.

Whole-mount staining and coronary arteriography. To visualize the coronary vessels in embryonic hearts, whole-mount staining was performed using antibodies against PECAM1, EMCN or LYVE1 as described previously ${ }^{64}$. Briefly, the hearts were isolated from E16.5 control and Pofut $1^{\mathrm{cKO}}$ embryos, and fixed in $4 \%$ PFA for $2-3 \mathrm{~h}$ at $4{ }^{\circ} \mathrm{C}$. The hearts were then blocked in $5 \%$ normal horse serum for $3 \mathrm{~h}$ at $4^{\circ}$ $\mathrm{C}$ and followed by incubation with primary antibodies at $4{ }^{\circ} \mathrm{C}$ overnight. The hearts were then washed for five times with PBS containing $0.5 \%$ TritonX-100 and incubated with biotinylated secondary antibodies at $4{ }^{\circ} \mathrm{C}$ overnight. The hearts were then washed for five times with PBS containing $0.5 \%$ TritonX-100 and incubated with streptavidin from $\mathrm{ABC}$ Kit (Vector Laboratories) at $4{ }^{\circ} \mathrm{C}$ overnight. The hearts were then washed for five times with PBS containing 0.5\% TritonX-100 
and developed color using DAB Kit (Vector Laboratories). In addition, coronary angiogram was performed to access the embryonic coronary arteries by injecting fluorescence dye into the left ventricle of beating hearts. The dye entered and labeled the coronary arteries through the coronary ostia (the coronary openings at the root of aorta). After injection the hearts were collected, fixed in 4\% PFA, and photographed using a Zeiss Discovery Microscope.

\section{Quantification of vessel density and VEGFR3 ${ }^{\text {high }}$ coronary angiogenic cells.} All quantifications were carried out using Image J. For quantifying the vessel area, images from PECAM1 antibody staining were used. The images from VEGFR3 antibody staining were used to quantify the number of VEGFR3+ cells. To quantify tip-like angiogenic cells, the heart sections were stained with PECAM1 and PECAM1-psoitive cells with microspikes were counted. The data were presented as the ratio of tip cells among total endothelial cells. Four hearts were analyzed for each experiment.

Spheroid angiogenic sprouting assay. Spheroid sprouting assay was performed as described previously ${ }^{65}$. Briefly, HUVEC (Life Technology) was cultured in M200 media. The cells were infected with Lentivirus carrying non-silence shRNA or POFUT1 shRNA and the infected cells were enriched by adding $2 \mu \mathrm{g} / \mathrm{ml}$ of puromycin to the media. Four thousand cells were cultured in round-bottom 96-well plates, which pre-coated with $0.8 \%$ agarose for spheroid formation. The spheroids were then embedded in fibrin gel and cultured in M200 media containing $10 \mathrm{ng} / \mathrm{ml}$ of VEGF for $48 \mathrm{~h}$. Then the spheroids were fixed in $4 \%$ PFA for 1 $\mathrm{h}$ and stained with phalloidin and imaged using EVOS microscope. The sprouting number was counted manually using Image J software. Eight spheroids were analyzed for each group in each experiment. The data from three independent experiments were subjected to statistic calculation.

Cell proliferation and apoptosis assays. Cell proliferation and cell apoptosis were determined using EdU and TUNEL assays, respectively, as described previously ${ }^{63}$. For cell proliferation assay, pregnant female mice were injected with EdU (Life Technology) through IP at a concentration of $100 \mathrm{mg} / \mathrm{kg}$. After a 2-hour pulse, the hearts were collected and processed for frozen sections as described above in Immunostaining Section. The serial sections crossing the whole heart were first stained with PECAM1 or VEGFR3 antibody followed by EdU staining with EdU imaging Kit (Life Technology) and counterstained with DAPI (Vector lab). The stained sections were photographed using a Zeiss Observer Z1 or Leica SP5 confocal microscope. EdU and PECAM1 or VEGFR3 double positive cells were counted using Image J and the data was presented as the ratio of EdU-positive cells among total PECAM1- or VEGFR3-positive cells. Three hearts were analyzed for each genotype. Apoptotic cells in the developing hearts of E14.5 embryos were visualized by TUNEL assay. The frozen sections of isolated hearts were prepared as described in the EdU study. Serial sections were first stained with PECAM1 antibody, followed by TUNEL assay by using DeadEnd ${ }^{m i}$ Fluorometric TUNEL System (Promega) and counterstained with DAPI. The stained sections were photographed using a Zeiss Observer Z1 or Leica SP5 confocal microscope.

RNA extraction and quantitative PCR (qPCR). To assess gene expression in mouse embryonic hearts, total RNA was extracted from embryonic hearts using the TRIzol solution (Life Technology) for reverse transcription to generate cDNAs with a Superscript II reverse transcriptase Kit (Life Technology). qPCR was performed using the Power SYBR Green PCR Master Mix (Life Technology) containing gene-specific primers (Supplementary Table 4). The relative expression of each gene was normalized to the expression of Gapdh and calculated using the 2 $-\Delta \Delta C{ }^{2}$ method. Biological replicates were performed using three individual samples of each genotype and technical triplicates were carried out for each run of qPCR. Student's $t$-test was used for statistic comparison between groups. $p<0.05$ was considered as significant.

Generation of Pofut1 knockout cells using CRISPR/Cas9. Lec1 CHO cells were transduced with Lentivirus carrying doxycycline-inducible Cas9 and selected with $20 \mu \mathrm{g} / \mathrm{ml}$ of puromycin. After 3 days, the cells were transduced with a second Lentivirus containing the guide RNA (gRNA) (5'-GGCGGCGCCCATGTCGGC GG-3'), which targets the second coding exon of Pofut1. After selection for 7 days in $25 \mu \mathrm{g} / \mathrm{ml}$ of blasticidin, resistant cells were maintained in the presence of both puromycin and blasticidin, and Cas9 expression was induced by addition of 500 $\mathrm{ng} / \mathrm{ml}$ doxycycline. After 7 days individual cell clones obtained by limiting dilution were analyzed by T7 endonuclease assay and DNA sequencing. Two independent clones having a deletion of two or four nucleotides in the

Cas9-gRNA site were shown to lack POFUT1 by western analysis with rabbit anti-bovine POFUT1 antibody ${ }^{16}$. Cells not treated with doxycycline were used as controls.

DLL4-Fc binding assay and NOTCH1 cell surface expression. Binding assays were performed as described previously ${ }^{66}$. Briefly, cells were washed with ligandbinding buffer (Hanks' buffered salt solution, $\mathrm{pH} 7.4,1 \mathrm{mM} \mathrm{CaCl}_{2}, 1 \%$ (w/v) bovine serum albumin, $0.05 \% \mathrm{NaN}_{3}$ ), fixed in $4 \%$ PFA in phosphate buffered saline without divalent cations (PBS-CMF) at RT for $10 \mathrm{~min}$ and stored in ligand binding buffer at $4^{\circ} \mathrm{C}$. Fixed cells $\left(1 \times 10^{6}\right)$ were incubated with $100 \mu$ ligand binding buffer containing $7.5 \mu \mathrm{g} / \mathrm{ml}$ DLL4-Fc or anti-hamster NOTCH1 antibody (R\&D Systems, AF5267, 1:50 in ligand binding buffer) at $4{ }^{\circ} \mathrm{C}$ for $1 \mathrm{~h}$. Soluble DLL4-Fc ligand was prepared as described previously ${ }^{66}$. After incubation, cells were washed twice with $0.5 \mathrm{ml}$ ligand binding buffer and incubated with phycoerythrin (PE)-conjugated goat anti-human IgG (Fc-specific) or Rhodamine Red-X-conjugated donkey anti-sheep IgG (1:100 in ligand binding buffer) at $4{ }^{\circ} \mathrm{C}$ for $30 \mathrm{~min}$ in the dark. The cells were then washed twice with $1 \mathrm{ml}$ of ligand binding buffer and analyzed in a FACS Calibur flow cytometer.

Cell migration assays. Cell migration studies were performed using HUVECs (Life technologies). $3 \times 10^{4}$ cells were plated onto 24 -well plates. For inhibition of NOTCH signaling, $20 \mu \mathrm{M}$ DAPT was added to the media, while DMSO was used as controls. For knockdown of POFUT1 or Dll4, the cells were infected with lentivirus carrying shRNA targeting POFUT1 or Dll4 respectively. The infected cells were enriched by puromycin selection. Knockdown efficiency was determined by qPCR. In cell migration assays, $10 \mathrm{ng} / \mathrm{ml}$ of VEGF120 was added to the media. The cells were subjected to live imaging using EVOS FL Auto Cell Imaging System. Analysis of individual cell tracks was performed using the DiPer Excel Macro ${ }^{67}$. For immunofluorescence, cells were plated onto glass coverslips. After 24-hour treatment, cells were fixed for $10 \mathrm{~min}$ with fixative solution (4\% paraformaldehyde, $0.1 \%$ Triton $\mathrm{X}$, and $0.15 \%$ glutaraldehyde in BRB80) and stained using rabbit anti-tubulin (Abcam, Ab18251), mouse anti-acetylated tubulin (Sigma, T6793) or actin (Alexa Fluor Phalloidin 488, A12379) antibodies for $1 \mathrm{~h}$ at RT. Then the cells were incubated with secondary antibodies conjugated with the Alexa 568 or 488 fluorescence dyes for $1 \mathrm{~h}$ at RT. Images were acquired using an EVOS FL Auto Cell Imaging System.

Statistical analysis. No statistical methods were used to predetermine sample size. Student's $t$-test (two-tailed) or one-way analysis of variance with Turkey's post-test was used for statistical difference between groups, assuming unequal variance from at least three independent experiments. Normality was assumed and variance was compared between groups. Sample size was determined based upon previous experience in the assessment of experimental variability. The investigators were not blinded to the group allocation during experiments and outcome assessment, unless stated otherwise. We chose the adequate statistic tests according to the data distribution to fulfill test assumptions. All numerical data were presented as mean $\pm \mathrm{SD}$ and $p$-value of $<0.05$ was considered as significant.

Data availability. The data supporting the findings of this study are available within the article and its Supplementary Information files and from the corresponding author on reasonable request.

Received: 11 January 2017 Accepted: 18 July 2017

Published online: 18 September 2017

\section{References}

1. Reese, D. E., Mikawa, T. \& Bader, D. M. Development of the coronary vessel system. Circ. Res. 91, 761-768 (2002).

2. Tomanek, R. J. Formation of the coronary vasculature during development Angiogenesis 8, 273-284 (2005).

3. Angelini, P. Coronary artery anomalies: an entity in search of an identity. Circulation 115, 1296-1305 (2007)

4. Haugen, O. A. \& Ellingsen, C. L. Coronary artery anomalies as a cause of sudden death in young people. Tidsskr. Nor. Laegeforen. 127, 190-192 (2007)

5. Fraisse, A. et al. Images in cardiovascular medicine. Myocardial infarction in children with hypoplastic coronary arteries. Circulation 101, 1219-1222 (2000).

6. Hauser, M. Congenital anomalies of the coronary arteries. Heart 91, 1240-1245 (2005).

7. $\mathrm{Wu}, \mathrm{B}$. et al. Endocardial cells form the coronary arteries by angiogenesis through myocardial-endocardial VEGF signaling. Cell 151, 1083-1096 (2012).

8. Red-Horse, K., Ueno, H., Weissman, I. L. \& Krasnow, M. A. Coronary arteries form by developmental reprogramming of venous cells. Nature 464, 549-553 (2010).

9. Katz, T. C. et al. Distinct compartments of the proepicardial organ give rise to coronary vascular endothelial cells. Dev. Cell 22, 639-650 (2012).

10. Jain, R. K. Molecular regulation of vessel maturation. Nat. Med. 9, 685-693 (2003).

11. Simons, M. \& Eichmann, A. Molecular controls of arterial morphogenesis. Circ. Res. 116, 1712-1724 (2015)

12. High, F. A. \& Epstein, J. A. The multifaceted role of Notch in cardiac development and disease. Nat. Rev. Genet. 9, 49-61 (2008).

13. Niessen, K. \& Karsan, A. Notch signaling in cardiac development. Circ. Res 102, 1169-1181 (2008) 
14. Guruharsha, K. G., Kankel, M. W. \& Artavanis-Tsakonas, S. The Notch signalling system: recent insights into the complexity of a conserved pathway. Nat. Rev. Genet. 13, 654-666 (2012).

15. Luca, V. C. et al. Structural biology. Structural basis for Notch1 engagement of delta-like 4. Science 347, 847-853 (2015).

16. Luca, V. C. et al. Notch-Jagged complex structure implicates a catch bond in tuning ligand sensitivity. Science 355, 1320-1324 (2017).

17. Roca, C. \& Adams, R. H. Regulation of vascular morphogenesis by notch signaling. Genes Dev. 21, 2511-2524 (2007).

18. Kofler, N. M. et al. Notch signaling in developmental and tumor angiogenesis. Genes Cancer 2, 1106-1116 (2011).

19. Liu, Z. et al. Notch1 loss of heterozygosity causes vascular tumors and lethal hemorrhage in mice. J. Clin. Invest. 121, 800-808 (2011).

20. Swift, M. R. \& Weinstein, B. M. Arterial-venous specification during development. Circ. Res. 104, 576-588 (2009).

21. Li, R. F. et al. Nr2flb control venous specification and angiogenic patterning during zebrafish vascular development. J. Biomed. Sci. 22, 104 (2015).

22. Gerhardt, H. et al. VEGF guides angiogenic sprouting utilizing endothelial tip cell filopodia. J. Cell Biol. 161, 1163-1177 (2003).

23. Hellstrom, M. et al. Dll4 signalling through Notch1 regulates formation of tip cells during angiogenesis. Nature 445, 776-780 (2007).

24. Suchting, S. et al. The Notch ligand delta-like 4 negatively regulates endothelial tip cell formation and vessel branching. Proc. Natl Acad. Sci. USA 104, 3225-3230 (2007).

25. Benedito, R. et al. The Notch ligands Dll4 and Jagged1 have opposing effects on angiogenesis. Cell 137, 1124-1135 (2009).

26. Ruhrberg, C. et al. Spatially restricted patterning cues provided by heparinbinding VEGF-A control blood vessel branching morphogenesis. Genes Dev. 16, 2684-2698 (2002).

27. Noseda, M. et al. Notch activation induces endothelial cell cycle arrest and participates in contact inhibition: role of p21Cip1 repression. Mol. Cell Biol. 24, 8813-8822 (2004).

28. High, F. A. et al. Endothelial expression of the Notch ligand Jagged1 is required for vascular smooth muscle development. Proc. Natl Acad. Sci. USA 105, 1955-1959 (2008)

29. Liu, H., Kennard, S. \& Lilly, B. Notch3 expression is induced in mural cells through an autoregulatory loop that requires endothelial-expressed Jagged 1. Circ. Res. 104, 466-475 (2009).

30. Volz, K. S. et al. Pericytes are progenitors for coronary artery smooth muscle. Elife 4, e10036 (2015).

31. del Monte, G. et al. Differential Notch signaling in the epicardium is required for cardiac inflow development and coronary vessel morphogenesis. Circ. Res. 108, 824-836 (2011).

32. Kerr, B. A. et al. Stability and function of adult vasculature is sustained by Akt/Jagged1 signalling axis in endothelium. Nat. Commun. 7, 10960 (2016).

33. Wang, Y. et al. Modification of epidermal growth factor-like repeats with $\mathrm{O}$-fucose. Molecular cloning and expression of a novel GDP-fucose protein O-fucosyltransferase. J. Biol. Chem. 276, 40338-40345 (2001).

34. Okajima, T., Xu, A. \& Irvine, K. D. Modulation of notch-ligand binding by protein O-fucosyltransferase 1 and fringe. J. Biol. Chem. 278, 42340-42345 (2003).

35. Shi, S. \& Stanley, P. Protein O-fucosyltransferase 1 is an essential component of Notch signaling pathways. Proc. Natl Acad. Sci. USA 100, 5234-5239 (2003).

36. Hang, C. T. et al. Chromatin regulation by Brg1 underlies heart muscle development and disease. Nature 466, 62-67 (2010).

37. Webb, D. J. et al. FAK-Src signalling through paxillin, ERK and MLCK regulates adhesion disassembly. Nat. Cell Biol. 6, 154-161 (2004).

38. $\mathrm{Hu}, \mathrm{Y}$. L. et al. FAK and paxillin dynamics at focal adhesions in the protrusions of migrating cells. Sci. Rep. 4, 6024 (2014).

39. Lauffenburger, D. A. \& Horwitz, A. F. Cell migration: a physically integrated molecular process. Cell 84, 359-369 (1996).

40. Tian, X. et al. Vessel formation. De novo formation of a distinct coronary vascular population in neonatal heart. Science 345, 90-94 (2014)

41. Sorensen, I., Adams, R. H. \& Gossler, A. DLL1-mediated Notch activation regulates endothelial identity in mouse fetal arteries. Blood 113, 5680-5688 (2009).

42. Bonini, S. A., Ferrari-Toninelli, G., Montinaro, M. \& Memo, M. Notch signalling in adult neurons: a potential target for microtubule stabilization. Ther. Adv. Neurol. Disord. 6, 375-385 (2013).

43. Charafeddine, R. A. et al. Fidgetin-like 2: a microtubule-based regulator of wound healing. J. Invest. Dermatol. 135, 2309-2318 (2015).

44. Maruta, H., Greer, K. \& Rosenbaum, J. L. The acetylation of alpha-tubulin and its relationship to the assembly and disassembly of microtubules. J. Cell Biol. 103, 571-579 (1986).

45. Xia, Y., Bhattacharyya, A., Roszell, E. E., Sandig, M. \& Mequanint, K. The role of endothelial cell-bound Jagged1 in Notch3-induced human coronary artery smooth muscle cell differentiation. Biomaterials 33, 2462-2472 (2012).
46. Zarkada, G., Heinolainen, K., Makinen, T., Kubota, Y. \& Alitalo, K. VEGFR3 does not sustain retinal angiogenesis without VEGFR2. Proc. Natl Acad. Sci. USA 112, 761-766 (2015).

47. Stanley, P. \& Okajima, T. Roles of glycosylation in Notch signaling. Curr. Top. Dev. Biol. 92, 131-164 (2010).

48. Lobov, I. B. et al. Delta-like ligand 4 (Dll4) is induced by VEGF as a negative regulator of angiogenic sprouting. Proc. Natl Acad. Sci. USA 104, 3219-3224 (2007).

49. Scehnet, J. S. et al. Inhibition of Dll4-mediated signaling induces proliferation of immature vessels and results in poor tissue perfusion. Blood 109, 4753-4760 (2007).

50. Tammela, T. et al. Blocking VEGFR-3 suppresses angiogenic sprouting and vascular network formation. Nature 454, 656-660 (2008).

51. Siekmann, A. F. \& Lawson, N. D. Notch signalling limits angiogenic cell behaviour in developing zebrafish arteries. Nature 445, 781-784 (2007).

52. Benedito, R. et al. Notch-dependent VEGFR3 upregulation allows angiogenesis without VEGF-VEGFR2 signalling. Nature 484, 110-114 (2012).

53. Ferrara, N. \& Kerbel, R. S. Angiogenesis as a therapeutic target. Nature 438, 967-974 (2005).

54. Tammela, T. et al. VEGFR-3 controls tip to stalk conversion at vessel fusion sites by reinforcing Notch signalling. Nat. Cell Biol. 13, 1202-1213 (2011).

55. Jiao, K. et al. An essential role of Bmp4 in the atrioventricular septation of the mouse heart. Genes Dev. 17, 2362-2367 (2003).

56. Cai, C. L. et al. A myocardial lineage derives from Tbx18 epicardial cells. Nature 454, 104-108 (2008).

57. Holtwick, R. et al. Smooth muscle-selective deletion of guanylyl cyclase-A prevents the acute but not chronic effects of ANP on blood pressure. Proc. Natl Acad. Sci. USA 99, 7142-7147 (2002).

58. Heidt, A. B. \& Black, B. L. Transgenic mice that express Cre recombinase under control of a skeletal muscle-specific promoter from mef2c. Genesis 42, 28-32 (2005).

59. Gustafsson, E., Brakebusch, C., Hietanen, K. \& Fassler, R. Tie-1-directed expression of Cre recombinase in endothelial cells of embryoid bodies and transgenic mice. J. Cell Sci. 114, 671-676 (2001).

60. Hozumi, K. et al. Delta-like 4 is indispensable in thymic environment specific for T cell development. J. Exp. Med. 205, 2507-2513 (2008).

61. Haiko, P. et al. Deletion of vascular endothelial growth factor C (VEGF-C) and VEGF-D is not equivalent to VEGF receptor 3 deletion in mouse embryos. Mol. Cell. Biol. 28, 4843-4850 (2008).

62. Moraes, F. et al. Endothelial cell-dependent regulation of arteriogenesis. Circ. Res. 113, 1076-1086 (2013).

63. Wang, Y. et al. Notch-Tnf signalling is required for development and homeostasis of arterial valves. Eur. Heart. J. 38, 675-686 (2015).

64. Wang, Y. et al. Endocardial to myocardial notch-wnt-bmp axis regulates early heart valve development. PLoS ONE 8, e60244 (2013).

65. Zheng, W. et al. Notch restricts lymphatic vessel sprouting induced by vascular endothelial growth factor. Blood 118, 1154-1162 (2011).

66. Stahl, M. et al. Roles of Pofut 1 and O-fucose in mammalian Notch signaling. $J$ Biol. Chem. 283, 13638-13651 (2008)

67. Gorelik, R. \& Gautreau, A. Quantitative and unbiased analysis of directional persistence in cell migration. Nat. Protoc. 9, 1931-1943 (2014)

\section{Acknowledgements}

We thank W. Lui for assistance with embryonic coronary arteriography. This work was supported by the National Institutes of Health/National Heart, Lung, and Blood Institute R01HL116997 and R01HL133120 (B.Z.), and the National Institute for General Medical Sciences R01GM106417 and the Albert Einstein Cancer Center grant NCI P0113330 (P. S.)

\section{Author contributions}

Y.W., B.W., P.L, D.Z., and B.W.: Performed most experiments. Z.Z.: Performed microCT. S.V.: Performed binding assays. R.C. and A.K. performed live imaging and quantification of microtubule expression. D.S., G.dMN., N.S., N.F., R.H., R.K., and P.S.: Helped data analysis. R.A. and K.A.: Provided the Cdh5CreERT2 and floxed Vegfr3 mouse line, respectively. Y.W. and B.Z.: Conceptualized the project. Y.W., B.W. and B.Z.: Supervised experiments. Y.W. and B.Z.: Wrote the manuscript. G.dMN., N.S., N.F., R.K., D.S., R.H., and P.S.: Critically edited the manuscript. All authors reviewed and approved the manuscript.

\section{Additional information}

Supplementary Information accompanies this paper at doi:10.1038/s41467-017-00654-w.

Competing interests: The authors declare no competing financial interests.

Reprints and permission information is available online at http://npg.nature.com/ reprintsandpermissions/ 
Publisher's note: Springer Nature remains neutral with regard to jurisdictional claims in published maps and institutional affiliations.

(c) (i) Open Access This article is licensed under a Creative Commons Attribution 4.0 International License, which permits use, sharing, Attribution 4.0 International License, which permits use, sharing, appropriate credit to the original author(s) and the source, provide a link to the Creative Commons license, and indicate if changes were made. The images or other third party material in this article are included in the article's Creative Commons license, unless indicated otherwise in a credit line to the material. If material is not included in the article's Creative Commons license and your intended use is not permitted by statutory regulation or exceeds the permitted use, you will need to obtain permission directly from the copyright holder. To view a copy of this license, visit http://creativecommons.org/ licenses/by/4.0/.

(C) The Author(s) 2017 